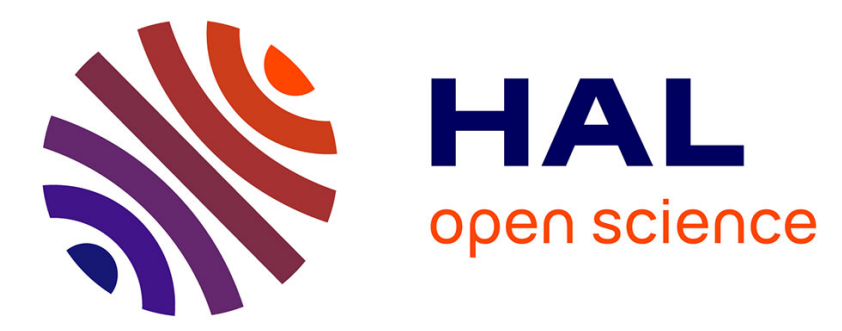

\title{
Le droit coutumier entre juridicisation et identité communautaire. De Sumatra à la France du Nord vers 1100
}

Charles de Miramon

\section{- To cite this version:}

Charles de Miramon. Le droit coutumier entre juridicisation et identité communautaire. De Sumatra à la France du Nord vers 1100. O. Condorelli, F. Roumy, M. Schmoeckel. Der Einfluss der Kanonistik auf die europäische Rechtskultur. Bd. 2 Öffentliches Recht., Böhlau, pp.81-114, 2011. halshs-00658055

\section{HAL Id: halshs-00658055 \\ https://shs.hal.science/halshs-00658055}

Submitted on 7 Apr 2017

HAL is a multi-disciplinary open access archive for the deposit and dissemination of scientific research documents, whether they are published or not. The documents may come from teaching and research institutions in France or abroad, or from public or private research centers.
L'archive ouverte pluridisciplinaire HAL, est destinée au dépôt et à la diffusion de documents scientifiques de niveau recherche, publiés ou non, émanant des établissements d'enseignement et de recherche français ou étrangers, des laboratoires publics ou privés. 


\title{
LE DROIT COUTUMIER ENTRE JURIDICISATION ET IDENTITÉ COMMUNAUTAIRE De Sumatra À la France du Nord vers i iOO*
}

\author{
Charles de Miramon
}

Le moulin de Beauvais, 6. - Penser le moment coutumier de la fin du $\mathrm{xI}^{e}$ au début du XII siècle, 13. - Maingaud, Guillaume et le droit coutumier, 17.

$\mathrm{C}^{\mathrm{N}}$ L'An 2000, le chapitre provincial de l'Association des conseils adat des villages du MinangE kabau, une province de louest de Sumatra, rendit une fatwa pour trancher un conflit entre le village de Lubuk Kilalang et la cimenterie de Padang. Le jugement expliquait que selon le droit coutumier (adat) Minangkabau, les biens communaux villageois ne pouvaient être pleinement aliénés. Il cassait dès lors la concession d'exploitation de calcaire à coraux accordée en 1972 à la cimenterie par le gouvernement central indonésien.

Franz et Keebet von Benda-Beckmann, anthropologues du droit et spécialistes de cette ethnie indonésienne célèbre pour être la plus grande société matrilinéaire au monde, observent que ce jugement est représentatif des modifications actuelles de P'équilibre en Indonésie entre le droit de type occidental (aujourd'hui plutôt dénommé transnational), le droit coutumier adat et le droit religieux islamique ${ }^{1}$. En 1998 , avec la chute de Soeharto, débute la reformasi, processus de transition démocratique dont l'un des aspects est la montée de revendications régionales et la remise en cause des concessions sur les ressources naturelles indonésiennes accordées par lancien régime; contrats qui avaient largement contribué à lenrichissement des familiers du dictateur. Le droit national indonésien héritier du droit colonial néerlandais considère que les espaces vierges sont des res nullius sur lesquelles l'État peut accorder des concessions. Cependant, selon l'adat de la région - ce mot équivalent du latin mos désigne l'ensemble des coutumes et des traditions des diverses régions et îles indonésiennes lensemble du territoire est divisé en zones de propriété clanique appartenant chacune à un

${ }^{*}$ Ch. de Miramon, «Le droit coutumier entre juridicisation et identité communautaire. De Sumatra à la France du Nord vers 1100 ", in : Der Einfluss des kanonischen Rechts auf die europäische Rechtskultur. Bd. 2 : Öffentliches Recht, éd. Fr. Roumy; O. Condorelli/M. Schmoeckel, Wien 2011, p. 81-114. La rédaction de cet article a été en partie réalisée à l'Institute for Advanced Study à Princeton en tant que membre Willis F. Doney. La pagination d'origine est indiquée en marge.

${ }^{1}$ Fr. von Benda-Beckmann, "Changing one is changing all : Dynamics in the Adat-Islam-State Triangle », Journal of Legal Pluralism and Unofficial Law 53-54 (2006), p. 239-270, URL : http: //www . jlp. bham . ac.uk. 
nagari (chef-lieu coutumier). On ne peut parler de res nullius. Le jugement de l'an 2000 renvoie à un vieux conflit de droit que nous voudrions quelque peu détailler, car il fournit une remarquable introduction aux questions que pose le droit coutumier.

Lorsqu'au cours du $\mathrm{xIx}^{\mathrm{e}}$ siècle, les Néerlandais investirent progressivement l'intérieur des îles et développèrent une économie de plantations, il fallut trouver des terres à exploiter. Le ministère des colonies chercha à donner un cadre juridique favorable à linvestissement et rédigea un code agraire qui édictait que les terres vierges étaient des res nullius susceptibles d'être allouées en concession. Très tôt pourtant, certains administrateurs coloniaux émirent des doutes sur lopportunité et la légalité du processus. En 1871, Arnold Verkerk Pistorius, indonologue autodidacte envoyé par ladministration coloniale étudier le Minangkabau, notait les particularités de la propriété foncière locale ${ }^{2}$. En fait, la politique agraire et l'opportunité de la 'modernisation' du régime de propriété foncière resta un lieu de conflits et de débats dans l'Indonésie coloniale puis indépendante ${ }^{3}$. D'un point de vue juridique, la question domaniale fut au centre du travail de Cornelis van Vollenhoven. Professeur de droit à Leyde au début du $\mathrm{xx}^{\mathrm{e}}$ siècle, père fondateur de lanthropologie juridique, Van Vollenhoven épousa les idées du courant dit 'éthique', favorable à une révision de la politique coloniale néerlandaise. Le colonisateur a un devoir moral envers les populations indigènes. Il ne faut pas, en particulier, imposer un ersatz de code de droit néerlandais sur les territoires indonésiens mais par une longue étude des coutumes locales faire émerger les principes organisateurs du adatrecht. Les idées de Van Vollenhoven rencontrèrent une triple réception. Ils trouvèrent une certaine application politique car les chrétiens-démocrates qui arrivent au pouvoir en 1901 furent les champions de la voie éthique. Leyde avait aussi à lépoque le monopole de formation en indonologie des administrateurs coloniaux et linfluence de ses professeurs sur les élites des Indes néerlandaises fut grande. Enfin, Van Vollenhoven créa une véritable école de l'adat qui se fixa pour tâche de collecter, d'analyser et de préparer la codification des différents droits coutumiers indonésiens. Le chef-d'œuvre de cette école fut la production de Pandectes de l'adat dont onze volumes parurent entre les deux guerres ${ }^{4}$.

En 1911, à Padang, le chef-lieu colonial du Minangkabau, le gouverneur de Sumatra occidental J. Ballot fit paraître à compte d'auteur une brochure sur la réforme agraire en cours. Ballot rend publique sa position consignée dans un rapport envoyé au ministère des colonies à La Haye. Il argumente que lon ne peut pas distribuer des terrains aux planteurs car ils appartiennent selon l'adat aux différents nagari. L'éthique Ballot reprend dans sa brochure un argument provenant d'un livre paru en 1885 de Willem Philip Scheuer ${ }^{5}$. Le communisme paysan des Nagari est comparable à linstitution médiévale du mark germanique. Scheuer s'inscrivait dans la lignée du juriste belge Émile De Laveleye qui, quelques années auparavant, avait dressé un tableau comparatiste mondial de la propriété foncière collective dans un ouvrage à succès véhiculant les fantasmagories germanistes alors à la mode ${ }^{6}$.

${ }^{2}$ A. W. P. V. Pistorius, «Studien over de inlandsche huishouding in de Padangsche bovenlanden », thèse de doct., Zaltbommel 1871.

${ }^{3}$ La littérature sur le sujet est abondante. Citons simplement : Fr. Durand, « La question foncière aux Indes Néerlandaises, enjeux économiques et luttes politiques (1619-1942) ", Archipel 58 (1999), p. 73-88.

${ }^{4}$ Pandecten van het adatrecht, Amsterdam 1914-1936.

${ }^{5}$ W. P. SCHeuer, Het grondbezit in de Germaansche mark en de Javaansche dessa, Rotterdam 1885.

${ }^{6}$ É. De Laveleye, De la propriété et de ses formes primitives, Paris $1891^{4}$. 
Ballot se place ainsi dans la stratégie argumentative du courant éthique qui articule deux arguments. D'un côté, il ne peut y avoir deux morales de laction selon que l'on exproprie aux Pays-Bas ou dans les colonies. L'expropriation doit s'incrire dans l'ordre juridique reconnu par les populations. D'autre part, le recours au passé mythifié de la communauté libre des paysans bataves primitifs, tout comme linvocation des figures tutélaires comme Grotius, remettaient en cause la légitimité du droit néerlandais de lépoque issu du Code napoléonien et de loccupation française. La glorification de l'adat correspondait aussi à des choix stratégiques du gouvernement local colonial. En 1908 avait eu lieu une jacquerie paysanne dans le Minangkabau suite à Pimposition par les Néerlandais de taxes indirectes. La situation apaisée, Ballot craignait d'édicter une mesure qui serait perçue comme discriminatoire par la population indigène et aurait pu provoquer de nouveaux troubles. Enfin, la situation politique locale mettait en compétition les chefs coutumiers traditionnels, les penghulu, et des mouvements islamistes conservateurs ou réformateurs. Ballot jouait la carte coutumière dans lespoir de freiner l'islamisation des élites indigènes et la montée des aspirations à lautonomie ${ }^{7}$.

Ballot trouva sur son chemin Nolst Trenité, homme fort de ladministration coloniale. Le gouverneur fut limogé et le nouveau code agraire de Sumatra fut promulgué en $1915^{8}$. G. J. Nolst Trenité devint ensuite l'un des enseignants d'un institut d'indonologie rival fondé dans les années vingt à l'université d'Utrecht avec lappui de Pindustrie pétrolière. Contre les 'éthiques' de Leyde, les 'réalistes' d'Utrecht argumentent que la construction de Van Vollenhoven est fragile, conduit à une fragmentation sans fin et à une incertitude juridique permanente. L'adatrecht ne peut répondre aux besoins des populations et doit avoir une place subordonnée dans l'ordre juridique local ${ }^{9}$. Après lindépendance, lordre juridique indonésien suivit les positions des réalistes et accorda une place limitée à l'adat.

À l'échec pratique de l'école de Leyde s'ajoute à partir des années 1990 une critique de méthode. On le sait, à cette époque, tant pour la coutume médiévale que pour les droits coutumiers des pays du Tiers-Monde se développent les argumentaires déconstructionnistes. Le droit coutumier serait une invention du juge médiéval ou de ladministrateur colonial qui par son regard préconçu d'expert transforme les fluctuantes traditions sociales en règles juridiques stables censées représenter un droit immémorial. On en vint à parler du « mythe de l'adat » et Peter Burns dans une monographie assez agressive contre Van Vollenhoven redéfinit l'adatrecht comme une simple construction de l'esprit d'un professeur exalté qui ne quitta presque jamais son bureau de Leyde et dont la connaissance de l'Indonésie était essentiellement livresque ${ }^{10}$.

${ }^{7}$ T. Abdullah, Schools and Politics : the Kaum Muda Movement in West Sumatra (1927-1933), Ithaca/New York 1971, p. 23-27.

${ }^{8}$ Laffaire est résumée dans J. S. KaHn, Constituting the Minangkabau. Peasants, Culture, and Modernity in Colonial Indonesia, Providence/Oxford 1993, p. 187-90.

${ }^{9} \mathrm{C}$. FAsSEur, «Colonial dilemma : Van Vollenhoven and the struggle between adat law and western law in Indonesia' ", in : The revival of tradition in Indonesian politics : the deployment of adat from colonialism to indigenism, éd. J. S. Davidson/D. Henley, Abingdon 2007, p. 50-67 déjà paru dans W. J. Mommsen/J. A. De Moor (éd.), European expansion and law. The encounter of European and indigenous law in 19th-and 20th-century Africa and Asia, Oxford/New York 1992.

${ }^{10}$ P. Burns, The Leiden Legacy. Concepts of Law in Indonesia, Leiden 2004 et la réponse de Fr. von BendABeckmann, Traditional Law in a Globalising World. Myths, Stereotypes, and Transforming Traditions, Van Vollenhoven Lecture 2008, Leiden 2008, URL : http: //www . vvi. leidenuniv.nl. 
Les évolutions juridiques récentes à Sumatra ne rentrent pourtant pas dans le schéma déconstructionniste. Le jugement de lan 2000 que nous avons mentionné en début d'article n'est pas un acte isolé. Il fait partie des évolutions politiques récentes que lon range sous Pétiquette du néo-traditionalisme. Elles ne sont pas spécifiques de l'Indonésie. On peut les retrouver sous des modalités diverses tant dans les combats indigénistes latino-américains que dans différents pays asiatiques ou africains. Elles combinent décentralisation politique, retour à des pouvoirs ou des sources de droit traditionnels et enfin judiciarisation par le bas qui conduit des communautés, parfois sous limpulsion d'ONG, à saisir les tribunaux pour faire valoir leurs droits. Dans les pays musulmans, un phénomène supplémentaire s'ajoute : Pimportance croissante du droit islamique dont les principes entrent parfois en conflit avec le droit coutumier. Pour les déconstructionnistes, il s'agit d'un feu de paille qui tient de la pantomime : les locaux jouent aux indigènes et réinventent une ritualité. Pour les tenants du pluralisme juridique, c'est, au contraire, la résurgence d'un phénomène occulté par la brutalité des gouvernements post-coloniaux.

Une approche historique est possible pour le Minangkabau relativement bien documenté. Elle permet de replacer le pluralisme juridique local dans une longue durée et de critiquer les explications déconstructionnistes. Tout d'abord l'adat, dans un sens juridique, existe avant larrivée des Occidentaux. Le mot vient du terme arabe el'ada qui désigne la coutume. La documentation concernant l'islamisation du Minangkabau est très peu fournie, mais une tradition peu sûre indique qu'en 1680 la royauté de type hindou — c'est-à-dire une institution plus cérémonielle que politique - se divise en trois : le roi de la terre, le roi de l'adat et le roi de la religion ${ }^{11}$. On peut penser que la cristallisation de l'adat de Minangkabau naît de la progression de la foi musulmane et de la créolisation de lislam. L'opposition entre la transmission traditionnelle clanique matrilinéaire et le droit de transmission patrilinéaire de la shari'ah serait le moyen par lequel l'adat marque sa différence et trouve sa légitimité. L'historien indonésien Taufik Abdullah a ainsi avancé la thèse que le conflit de droit serait le moteur de lhistoire des Minangkabau ${ }^{12}$. Si on sait peu de la période pré-islamique, les mythes locaux ainsi que certaines institutions montrent que l'adat n'est pas perçu comme une totalité mais comme en système clivé par moitié. Dès avant Pislamisation s'opposent l'adat du système royal et celui des nagari, Padat des Minangkabau et celui des autres peuples indonésiens ${ }^{13}$. Les différents clans des différents villages dépendent d'un des deux systèmes d'adat qui different sur la place de la composition et de la procédure pénale. Par la suite, lopposition majeure concerne la shari'ah et l'adat. Au début du xix ${ }^{\mathrm{e}}$ siècle, la guerre des Padris vit s'affronter deux factions, les partisans de l'adat et les padris inspirés par le wahhabisme qui voulaient imposer le droit islamique et en particulier la fin de la propriété matrilinéaire ${ }^{14}$. Les Néerlandais intervinrent et prirent alors contrôle du Minangkabau. Un autre mouvement d'opposition dont lune des clés de lecture est le clivage islam / adat se déroula dans les années $1920^{15}$. À la sortie de ces

\footnotetext{
${ }^{11}$ P. E. J. de DE Jong, Minangkabau and Negri Sembilan. Socio-Political Structure in Indonesia, Den Haag 1980, p. 101-102.

${ }^{12}$ T. Abdullah, « Adat and Islam : an examination of conflict in Minangkabau », Indonesia 2 (1966), p. 1-24.

${ }^{13}$ T. Kato, «Change and Continuity in the Minangkabau Matrilineal System », Indonesia 25 (1978), p. 1-16.

${ }^{14} \mathrm{Chr}$. Dobbin, Islamic Revivalism in a Changing Peasant Economy : Central Sumatra, 1784-1847, London/Malmö 1983.

${ }^{15}$ T. Abdullah, The Kaum Muda Movement (cf. n. 7).
} 
conflits, les élites locales prirent conscience qu'il fallait faire cohabiter droits occidental, islamique et coutumier. Le jugement de lan 2000 est un exemple de fusion de ces influences. Il est donné par un tribunal qui est le résultat d'une réforme administrative 'à loccidentale'; il se dénomme fatwa pour donner une coloration religieuse à la décision et reprend un vieux principe de l'adat local.

Que fait un historien du droit médiéval dans les jungles humides de Sumatra? Sur un sujet aussi glissant que le droit coutumier et sur lequel on a tant écrit, il s'agit avant tout de se déciller le regard. Une première leçon est que la perspective finaliste européenne de labsorption des droits coutumier et canonique par le Léviathan étatique n'est pas universelle. L'exemple indonésien montre que d'autres scénarios sont possibles. La deuxième leçon qu'il faut retenir est le caractère identitaire de l'adat. Le droit coutumier existe par ce qu'il est perçu comme différent d'un autre droit. L'adat nait de la friction avec le droit islamique et accessoirement du droit occidental. Si lon se tourne vers le Moyen Âge occidental, la question n'est donc pas tellement de savoir s'il existe ou non un droit oral immémorial qui n’atteindrait lécrit que tardivement. Il s'agit plus de se demander comment au sein d'un processus de juridicisation, une identité coutumière naît. Cette perspective est différente de celle qui perçoit le droit coutumier uniquement comme un artefact du droit savant comme une norme sinon rejetée du moins douteuse et cantonnée dans un rôle subsidiaire ${ }^{16}$. Pour étudier la naissance de cette identité coutumière dans la France médiévale, nous nous intéresserons à la période charnière des $\mathrm{XI}^{\mathrm{e}}$ et $\mathrm{XII}^{\mathrm{e}}$ siècles, période où la théorie romano-canonique des sources du droit n'est pas encore dominante et où les jeux sont encore ouverts. Cet article continue ainsi celui paru dans le volume précédent de cette série et procède de la même méthode : confronter deux études de cas avec des textes théoriques contemporains : les commentaires sur le De inventione de Maingaud et Guillaume de Champeaux ${ }^{17}$. Les deux exemples d'invention précoce de la coutume que je vais étudier dans une perspective tant d'histoire sociale que juridique proviennent de Beauvais. Le premier dossier concerne un moulin et oppose les burguenses de Beauvais et le chapitre cathédral. Il nous est connu par deux sources - une notice judiciaire et une lettre d'Yves de Chartres - qui ne sont pas concordantes. Le deuxième exemple est un conflit réglé lors d'un plaid autour d'une maison entre une famille locale et les chanoines réguliers de Saint-Quentin.

\footnotetext{
${ }^{16}$ Voir ainsi A. Gouron, "Aurore de la coutume », Recueil de mémoires et travaux publié par la Société d'histoire du droit et des institutions des anciens pays de droit 14 (1988), p. 181-87 et plus généralement les différents articles regroupés dans IDEM, Droit et coutume en France aux XII et XIII siècles, Aldershot 1993 où cette position est défendue avec beaucoup de brio.

${ }^{17} \mathrm{Ch}$. de Miramon, «Guillaume de Champeaux et la règle de droit des personnes. Droit et communautés urbaines dans la France du Nord au tournant du xi ${ }^{\mathrm{e}}$ et du xII siècle ", in : Der Einfluss des kanonischen Rechts auf die europäische Rechtskultur. Bd. 1 : Zivil- und Zivilprozessrecht, éd. Fr. Roumy ; M. Schmoeckel/O. Condorelli, Wien 2009, p. 33-65.
} 


\section{Le MOUlin de Beauvais}

En 1099, les burguenses de Beauvais se disputèrent avec les chanoines de la cathédrale autour d'un moulin ${ }^{18}$. Les chanoines se plaignent que les bourgeois obstruent le cours de la rivière en amont de leur moulin qui leur répondent qu'ils ont un libre usage du cours d'eau. Nous sommes à laube du développement urbain de Beauvais et le petit nombre de documents disponibles ne permet pas de dépeindre avec précision le contexte. Cependant, ce conflit est révélateur des évolutions juridiques de l'époque. Il mérite que l'on s'y arrête ${ }^{19}$.

Qui sont ces bourgeois et où se trouve ce moulin? La notice judiciaire ne donne aucune indication toponymique. Une lettre d'Yves de Chartres sur laquelle nous reviendrons indique que la marche du moulin était perturbée par un pont et les déchets des tanneurs (l'édition parle cependant des teinturiers $)^{20}$. Pour éclaircir le sens de ces allusions, il faut reprendre la question du développement urbain au $\mathrm{xI}^{\mathrm{e}}$ siècle.

Comme pour d'autres villes du Nord de la France, Beauvais est selon l'heureuse expression d'André Guillerme, une 'petite Venise ${ }^{21}$. Les travaux collectifs d'aménagement des rivières forment la base de lurbanisme. Ils permettent le développement d'activités industrielles qui utilisent P'énergie hydraulique ou le flux d'eau (moulins, tannerie, teinturerie, industrie de la laine $)^{22}$. Dans le cas de Beauvais, le Thérain est canalisé et dérivé en plusieurs canaux urbains afin d'offrir un débit constant favorable à la moulinerie et à Partisanat ${ }^{23}$. À partir du milieu du

${ }^{18}$ Sur les moulins, voir la récente synthèse de M. Arnoux, « Les moulins à eau en Europe occidentale ( $\mathrm{Ix}^{\mathrm{e}}-$ $\mathrm{XII}^{\mathrm{e}}$ siècle). Aux origines d'une économie institutionnelle de l'énergie hydraulique ", in : L'acqua nei secoli altomedievali, (Settimane di studio, 55), Spoleto 2008, p. 693-746 et deux colloques récents : P. Galetti/P. Racine (éd.), I mulini nell'Europa medievale, Bologna 2003 et M. Mousnier (éd.), Moulins et Meuniers dans les campagnes européennes (IX $-X V I I I^{e}$ siècle), Toulouse 2002. Les travaux récents montrent que les moulins sont plus présents dans les campagnes du Haut Moyen Âge qu'on le pensait naguère.

${ }^{19}$ Sur Beauvais à cette époque, les deux ouvrages fondamentaux sont : L.-H. LABANDE, Histoire de Beauvais et de ses institutions communales jusqu'au commencement $d u X V^{e}$ siècle, Paris 1892 et O. Guyotjeannin, Episcopus et comes. Affirmation et déclin de la seigneurie épiscopale au nord du Royaume de France (Beauvais - Noyon, $X^{e}-$ début XIII siècle), Genève / Paris 1987.

${ }^{20}$ Yves de Chartres, Epist. 77 : « Nunc vero impedimentis pontium et tinctorum sordibus impeditum molendi amisit officium » (PL 162, col. 98 qui reprend l'édition de François Juret, Paris, 1610, p. 154). La teinturerie est certes polluante mais cette pollution est gênante pour ceux qui ont besoin d'une eau claire car ils doivent laver de la laine, des draps ou pêcher. On a du mal à voir en quoi elle gêne un moulin. En revanche, d'autres textes mentionnent des poteaux et un cens des peaux à proximité du moulin (cf. infra). On peut donc penser que le flux d'eau arrivant au moulin était perturbé par le 'travail de la rivière' des tanneurs, c'est-à-dire la nécessité de laisser les peaux tremper dans la rivière pour les laver à différentes étapes de leur transformation en cuir. Un texte de 1454 décrit avec précision le travail du sergent des eaux, lofficier épiscopal chargé de la police de la rivière. Il y est mentionné que chaque tanneur a le droit à cinq pieux pour son travail à condition que les peaux pendent le long du courant et non pas en travers (L.-H. Labande, Histoire de Beauvais (cf. n. 19), p. 333). C'est sans doute ce travail de la rivière qui était en jeu en 1099. Je propose donc de corriger tinctorum en tannorum. La mention de teinturerie est une erreur soit de l'édition Juret, soit d'Yves, soit de son secrétaire.

${ }^{21}$ A. Guillerme, Les Temps de l'eau. La cité, l'eau et les techniques. Nord de la France, fin III ${ }^{e}$ début XIX ${ }^{e}$ siècle, Seyssel 1983, p. 68-73.

${ }^{22}$ Voir en général Ph. Bourgès, «Une réussite économique : les moulins hydrauliques de Beauvais à la fin du XII ${ }^{\mathrm{e}}$ siècle ", in : I mulini nell'Europa medievale, éd. P. GAletti/P. Racine, Bologna 2003, p. 203-216 malheureusement peu précis sur la chronologie et la localisation des moulins.

${ }^{23}$ Guillerme fait Phypothèse, sans beaucoup d'indices, que les dérivations urbaines du Thérain seraient lœuvre de l'évêque Roger au xi ${ }^{\mathrm{e}}$ siècle qui aurait creusé d'anciennes voies romaines (A. Guillerme, Les Temps de l'eau (cf. n. 21), p. 70-71. Pour d'autres (O. Guyotjeannin, Episcopus et comes (cf. n. 19), 30, n. 140), le réseau 


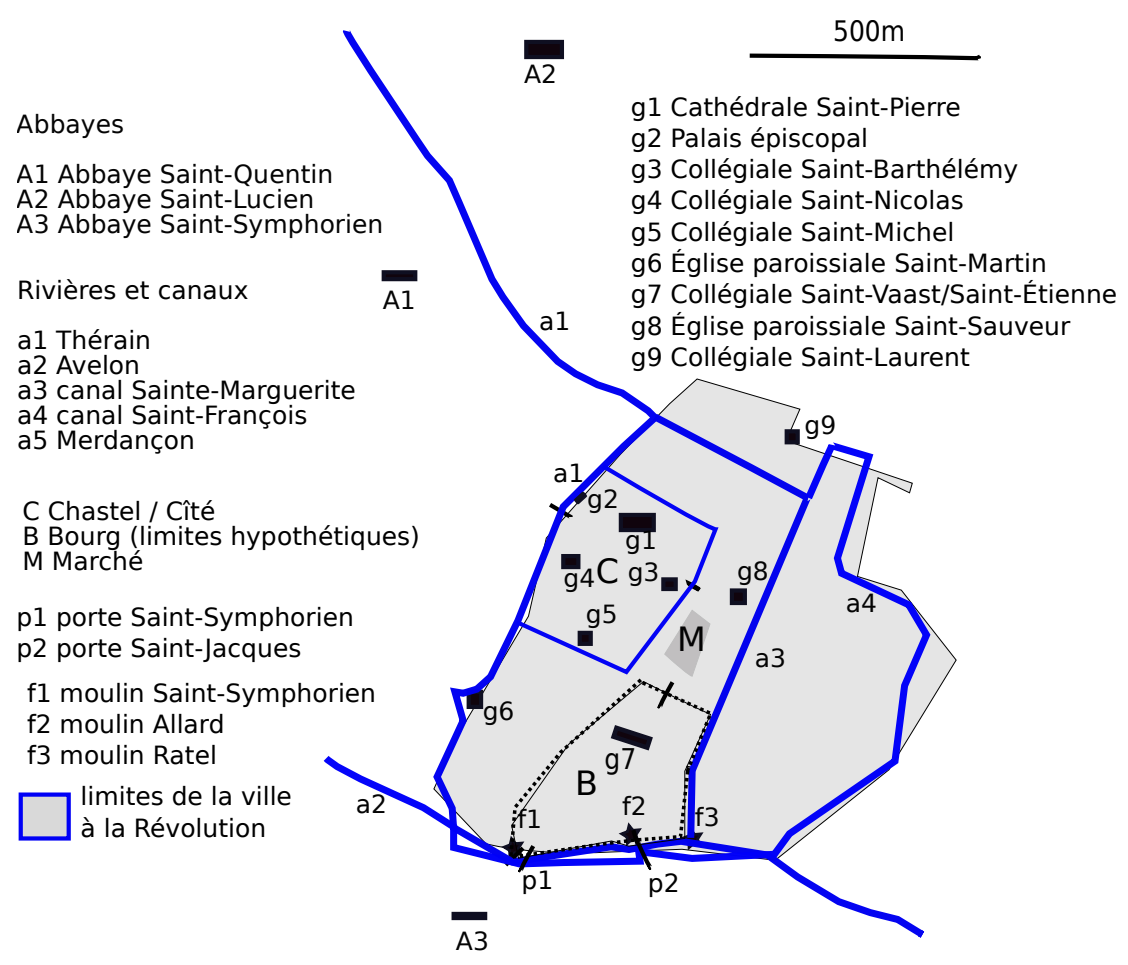

FIG. 1 : Beauvais vers 1100

$\mathrm{XII}^{\mathrm{e}}$ siècle, les moulins se multiplient, particulièrement les moulins à foulon; Beauvais étant un centre de draperie.

Lurbanification de Beauvais suit une facture classique. Elle se découvre dans le plan cicontre $^{24}$. Une ancienne cité antique de petite taille, entourée d'une muraille et d'un fossé défensif, rassemble la cathédrale, le quartier canonial, la ville ecclésiastique et militaire. L'expansion marchande et artisanale se déroule en dehors de lurbs. On y trouve un marché et des noyaux de population organisés autour des églises ou des établissements religieux suburbains. Une deuxième enceinte, sans doute érigée durant le $\mathrm{xII}^{\mathrm{e}}$ siècle, fixera définitivement la forme de lagglomération.

Pour le $\mathrm{xI}^{\mathrm{e}}$ siècle, lacte le plus instructif sur le développement de Beauvais est une charte de 1072 par laquelle l'évêque Guy refonde la collégiale de Saint-Étienne-Saint-Vaast. Guy explique que Saint-Étienne-Saint-Vaast se trouve dans un bourg (in burgo) et qu'elle est la mère d'églises tant dans la banlieue (in suburbio) que dans la ville (in urbe). Cet acte semble indiquer

est d'origine antique mais Desachy (Br. Desachy, « Le site archéologique de Beauvais (Oise). Éléments de présentation topographique et chronologique ", Revue archéologique de Picardie 3-4 (1991), p. 11-43, p. 32) maintient l'hypothèse du xI $\mathrm{e}^{\mathrm{e}}$ siècle. Les témoignages archéologiques ne semblent pas indiquer d'habitat urbain à proximité de ces canaux avant le Moyen Âge central. Pour Guillerme la réalisation des canaux correspond à une opération de lotissement par laquelle on trace les axes et les limites du développement de la ville médiévale. Cette position me semble pertinente.

${ }^{24} \mathrm{La}$ carte reprend le travail de Desachy cité à la note précédente en y ajoutant mes propres hypothèses sur le bourg Saint-Étienne. 
lexistence d'un bourg autour de Saint-Étienne, une hypothèse acceptée par plusieurs historiens locaux ${ }^{25}$. Un autre document intéressant provient de Saint-Quentin de Beauvais. Dans leur obituaire, les chanoines réguliers énumèrent les donations de leur fondateur, lévêque Guy. Il leur a offert en particulier «les dîmes de ses moulins situés devant la porte, le cens des peaux, la porte de Saint-Symphorien et la porte de Saint-Jacques ${ }^{26}$ ». Les trois donations forment un groupe après un premier ensemble concernant l'entourage immédiat du monastère; puis viennent les dons de prébendes. Si lon suit la logique de l'énumération, il s'agit de revenus provenant de lieux voisins. Le cens des peaux renvoie aux tanneurs beauvaisiens dont on peut localiser lactivité plus tard au Moyen Âge sur les canaux Sainte-Marguerite et Saint-François. Les désignations des portes suggèrent que lon ne parle pas ici des portes de la cité. La porte Saint-Symphorien désigne ce qui sera nommé plus tard la porte Saint-Jean, une porte (ensuite fortifiée) et un pont qui permet de traverser le Thérain pour se rendre à labbaye Saint-Symphorien. La porte Saint-Jacques est sans doute la future porte de Paris, autre lieu légèrement en aval pour traverser la rivière en direction du futur faubourg SaintJacques ${ }^{27}$. Qu'il y ait des moulins à proximité de ces portes est logique. Elles se situent peu après la confluence de l'Avelon et du Thérain; le cours de la rivière y est puissant et régulier, propice à la meunerie. Lacte de Saint-Quentin nous indique donc que vers 1070, il y avait un mur défensif percé de deux portes qui sera par la suite incorporé dans la deuxième muraille urbaine. Ce premier mur ne doit pas être autre que celui qui devait entourer au $\mathrm{xI}^{\mathrm{e}}$ siècle le bourg Saint-Étienne et dont les contours épousaient vraisemblablement les limites paroissiales telles qu'elles sont connues pour le début de l'Époque moderne ${ }^{28}$. Le bourg aura une vie brève. Dans une bulle de confirmation des possessions de Saint-Quentin en 1108-1110, il est parlé « des moulins de l'évêque qui sont devant la porte de la cité ", indice que les nouvelles limites urbaines incorporant les ensembles anciens sont désormais perçues par tous ${ }^{29}$. L'incendie du suburbium en 1111 a certainement joué un rôle dans la fusion des ensembles ${ }^{30}$. Cette existence fugace imprimera pourtant sa marque dans la mémoire collective. C'est dans le cimetière de Saint-Étienne que se trouve 'la commune', c'est-à-dire le tertre, ensuite aménagé en tribune où se déroulaient les élections municipales et où le nouveau maire prêtait serment $^{31}$. Saint-Étienne est léglise des marchands et son clocher conserve la cloche commu-

\footnotetext{
${ }^{25} \mathrm{Br}$. Desachy, «Le site archéologique de Beauvais » (cf. n. 23), p. 29 ; A. Henwood-Reverdot, L'Église SaintÉtienne de Beauvais : histoire et architecture, Beauvais 1982, p. 6.

${ }^{26}$ «Dedit etiam nobis parrochiam Sancti Ypoliti, prebendam Sancti Petri, prebendam Sancti Vedasti, decimationem molendinorum suorum ante portam ; censum pellium, portam Sancti Symphoriani et portam Sancti Jacobi » (H. Omont, « Donations d'Ives de Chartres et de l'évêque Gui à labbaye de Saint-Quentin de Beauvais ", Bibliothèque de l'École des Chartes 66 (1905), p. 631-632, p. 632).

${ }^{27}$ Cependant, P'église Saint-Jacques n'est attestée qu'au xıII siècle.

${ }^{28}$ V. Lhuillier, La Paroisse et l'Église Saint-Étienne de Beauvais, Beauvais 1896, p. 57-66.

${ }^{29}$ Papsturkunden in Frankreich. Neue Folge. 7. Band, Nördliche Île-de-France und Vermandois, éd. D. Lohrmann, Göttingen 1976, $\mathrm{n}^{\circ} 22$; même formulation dans lacte de 1111 de Louis VI ( Recueil des actes de Louis VI roi de France (1108-1137), éd. J. Dufour, Paris 1992-94, n 53). Cependant, en 1108 l'évêque Geoffroy donne encore au chapitre sex bolengarios de burgo Belvacensi (Ibid., $\mathrm{n}^{\circ} 83$ ).

${ }^{30}$ MGH SS 6, p. 462.

${ }^{31}$ L. Carolus-BARré, «La Commune ou la Tribune aux harangues dans lancien cimetière de l'église Saint-Étienne de Beauvais ", Mémoires de la Société académique de l'Oise 12 (1883), p. 763-771. La documentation sur le sujet remonte à la fin du Moyen Âge.
} 
nale ${ }^{32}$. Il y a ainsi une certaine superposition à Beauvais entre l'esprit communal et les libertés de laître de Saint-Étienne.

Il existait donc dans la deuxième moitié du xi ${ }^{\mathrm{e}}$ siècle un bourg Saint-Étienne à Beauvais. Ceci conduit à proposer une nouvelle interprétation du terme burguenses que lon trouve dans la notice judiciaire. En 1100, nous sommes à l'époque où le vocable 'bourgeois' fait sa mue vers le sens que nous lui connaissons de citoyen ${ }^{33}$. Il semble pourtant que c'est le sens traditionnel qu'il faut retenir ici : les burguenses sont les habitants du bourg Saint-Étienne ${ }^{34}$.

On comprend dès lors mieux les ressorts du procès autour du moulin si on le replace dans un conflit de plus longue durée pour le contrôle du suburbium de Beauvais dont lun des aspects est la compétition entre le chapitre l'évêque et les habitants du bourg pour la possession des moulins et le partage de ses différents revenus (droits de mouture, dîme, obligation de certains boulangers de moudre dans un moulin spécifique). Guy avait certainement une dent contre certains chanoines cathédraux. Évêque réformateur, il modifie le paysage ecclésiastique du suburbium en fondant Saint-Quentin (les chanoines réguliers ayant une fonction sinon pastorale du moins liturgique qui dépasse le monastère et ses stricts alentours) et la canoniale de Saint-Étienne qui remplace une église sous personat ${ }^{35}$. À chaque fois, l'évêque abaisse son chapitre cathédral. Il offre une prébende du chapitre à Saint-Quentin et une autre à Saint-Étienne. Saint-Étienne se voit attribuer un rôle dans la direction du réseau paroissial urbain. Lors de la dédicace de Saint-Quentin, il humilie publiquement le trésorier du chapitre cathédral accusé d'accaparer la moitié de la dîme ${ }^{36}$. Il déplace ensuite les reliques de sainte Romaine de la cathédrale à Saint-Quentin ${ }^{37}$. Lacte de fondation en 1078 de Saint-Nicolas, une canoniale intra-muros, contient une nouvelle attaque contre les chanoines de la cathédrale soupçonnés d'acheter leurs prébendes ${ }^{38}$. Il leur est enjoint de choisir pour nouveau trésorier, un clerc 'utile et prudent', élu sans simonie qui doit en particulier organiser une école pour les enfants ${ }^{39}$. Les chanoines de la cathédrale ne restèrent pas immobiles devant cette hosti-

\footnotetext{
${ }^{32}$ A. Henwood-Reverdot, L'Église Saint-Étienne de Beauvais (cf. n. 25), p. 19.

${ }^{33}$ G. Duby (éd.), Histoire de la France urbaine, Paris 1980, t. 2, p. 103-105.

${ }^{34}$ Sur le sens classique, voir Tr. Endemann, Markturkunde und Markt in Frankreich und Burgund von 9. bis 11. Jahrhundert, Konstanz/Stuttgart 1964, chap. 6.

${ }^{35}$ Sur Guy (qui mériterait une étude plus approfondie) : O. GuYotjeannin, Episcopus et comes (cf. n. 19), p. 7072, L.-H. Labande, Histoire de Beauvais (cf. n. 19), p. 48-52 et C. Mews, "St Anselm, Roscelin and the See of Beauvais ", in : Anselm. Aosta, Bec and Canterbury, éd. D. E. Luscombe/G. R. Evans, Sheffield 1996, p. 106-119 repris dans IDEm, Reason and belief in the age of Roscelin and Abelard, Aldershot 2002. Le personat de SaintÉtienne est donné par le chantre Roscelin et Névelon de Compiègne en 1072. Constant Mews a proposé d'identifier ce Roscelin avec le philosophe Roscelin de Compiègne (C. Mews, "St Anselm, Roscelin and the See of Beauvais » (cf. n. 35), p. 111-112). Nous savons que Roscelin de Compiègne est présent à Beauvais en 1090 à l'époque de sa controverse avec saint Anselme sur la Trinité. Cependant, il n'est pas chantre à la cathédrale. En effet, dès 1078 (acte mentionné infra, n. 39 ) le chantre est un certain Drogon.

${ }^{36}$ L.-H. Labande, Histoire de Beauvais (cf. n. 19), p. 260-261.

${ }^{37}$ AA. SS., Oct. II, p. $137-140$.

${ }^{38}$ Saint Anselme accusera un peu plus tard le chapitre d'héberger des chanoines en concubinage notoire qui lèguent leurs prébendes à leurs enfants (Epist. 126, Anselmus Cantuariensis, Opera omnia, éd. Fr. Schmitt, Stuttgart 1968, t. 3, p. 267-268).

39 «Et quoniam pullulante cupiditate omnium malorum radice perversus in Ecclesia mos sibi locum usurpavit ut quidam canonici in pluribus locis praebendas acquirant et pro sua absentia ibi vicarios apponant. Ideo decrevimus ne aliquis clericus in alia Ecclesia attitulatus ibi praebendam usurpet nec alicui ibidem prius attitulato et postea propter inanis gloriae cupiditatem volenti ad aliam ecclesiam transmeare prior prebenda permaneat
} 
lité. Ils provoquèrent sans doute les troubles qui contraignirent Guy à s'enfuir de son siège épiscopal quelque temps. Plusieurs années plus tard, ils furent sans doute responsables de Paccusation de simonie portée contre Guy, arroseur arrosé, qui le conduisit à se réfugier à Cluny où il mourut ${ }^{40}$. De plus, à une époque indéfinissable (durant ou après l'épiscopat de Guy), les chanoines forgèrent une fausse charte incorporant du matériel carolingien. Elle détaillait en particulier leurs possessions sises dans le suburbium ${ }^{41}$. À travers cette forgerie qui ne mentionne évidemment aucun autre établissement ecclésiastique périurbain, le but des chanoines de la cathédrale est clair : asseoir lantiquité de leur domination sur le suburbium et s'armer contre les prétentions des nouveaux arrivants. L'acte mentionne ainsi que l'évêque carolingien Eudes aurait légué au chapitre deux moulins dans le suburbium ${ }^{42}$. La mémoire des chanoines étaient assez plastique pour imaginer que les moulins auraient été aussi donnés par l'évêque, plus tardif, Hervé ${ }^{43}$.

Cela fait beaucoup de moulins! Il ne faudrait pourtant pas multiplier les roues à aubes sur le Thérain. Au xII ${ }^{\mathrm{e}}$ siècle, lorsque la documentation devient plus précise, il n'y a que deux, peut-être trois, moulins à cet endroit de la rivière ${ }^{44}$. Cette configuration restera inchangée jusqu'à la Révolution ${ }^{45}$.

Au tournant du $\mathrm{xI}^{\mathrm{e}}$ et du $\mathrm{XII}^{\mathrm{e}}$ siècle, la situation va se retourner en faveur des chanoines. Les nombreux conflits lors des successions épiscopales expliquent sans doute le retour en puissance du chapitre cathédral courtisé par tous lors des élections ${ }^{46}$. En tout cas lors du court épiscopat d'Anseau à lextrême fin du xi ${ }^{\mathrm{e}}$ siècle, les chanoines obtiennent non seulement un jugement favorable dans laffaire du moulin mais aussi un important privilège de l'évêque leur accordant le droit de boulangerie dans la banlieue de Beauvais et la justice banale avec possibilité d'excommunier les fauteurs de troubles ${ }^{47}$. Ils vont même essayer de récupérer des droits sur le suburbium de Saint-Quentin. Yves de Chartres doit même envoyer une autre lettre indignée à l'évêque Anseau pour se plaindre du doyen Hugues et rappeler les donations de Guy a son ancienne abbaye ${ }^{48}$.

quia plerumque videmus contingere, ut ex huiusmodi licentia perversa et vicariorum vel potius mercenariorum negligentia desertae a servientibus fiant ecclesiae. [...] Qua propter etiam decernimus ut canonici beati Petri in praefata ecclesia prudentem et utilem clericum absque ullo pretio vel mercede retributionis in officio thesaurarii constituant et gratis prebendam tribuant qui in disciplina ad servitium ecclesiae pueros edoceat et ipsam ecclesiam luminaribus, tectura et coeteris necessariis restauret » (P. Louvet, Histoire et antiquitez du païs de Beauvaisis, Beauvais 1631, p. 690-91).

${ }^{40}$ O. Guyotjeannin, Episcopus et comes (cf. n. 19).

${ }^{41}$ IDEM, «Un témoignage falsifié des possessions primitives de P'église cathédrale de Beauvais : examen et tradition ", Francia 13 (1985), p. 687-694 qui pense que lacte est composé au xi ${ }^{\mathrm{e}}$ siècle.

${ }^{42}$ Ibid., p. 693.

${ }^{43}$ « vi. id. april. Eodem die, obiit Herveus episcopus, qui dedit nobis mansos XIII in suburbio nostre civitatis et duo molendino » (L.-H. Labande, Histoire de Beauvais (cf. n. 19), 22, n. 1 d'après un obituaire aujourd'hui disparu). L'information provient d'une mauvaise lecture du faux d'Eudes.

${ }^{44}$ Le moulin du Ratel est mentionné dans un acte de 1109; le moulin Allard apparaît dans la documentation vers le milieu du XII ${ }^{\mathrm{e}}$ siècle (Ibid., 217, n. 6).

${ }^{45}$ Voir la notice d'Henri Fromage sur les moulins à Beauvais dans le numéro spécial : «Beauvais en 1789 : le bâti de la ville ", Bulletin du Groupe d'étude des monuments et cuvres d'art de l'Oise et du Beauvaisis 35-36 (1989).

${ }^{46}$ Par exemple C. Mews, «St Anselm, Roscelin and the See of Beauvais» (cf. n. 35).

${ }^{47}$ P. Louvet, Histoire et antiquitez du païs de Beauvaisis (cf. n. 39), p. 290-291.

${ }^{48}$ Yves de Chartres, Epist. 281 (PL 162, col. 282). Cette lettre pose un problème d'histoire textuelle. Elle est adressée à Anseau († 1100) mais est incorporée dans une section du registre qui regroupe les lettres écrites en 
En un mot, le conflit sur le moulin de 1099 doit se replacer dans un faisceau de circonstances : les blessures occasionnées par l'épiscopat de Guy, la compétition entre le chapitre cathédral et les nouvelles collégiales pour le contrôle paroissial et banal de la ville en développement et enfin les conflits entre le chastel aristocratique et ecclésiastique et le bourg marchand.

Ce qui fait pourtant le sel du dossier ce n'est pourtant pas son aspect socio-économique, mais juridique. Pour l'histoire du droit, la qualité des sources est exceptionnelle. La notice du jugement est un document bref mais remarquable connu par une édition du xvir ${ }^{\mathrm{e}}$ siècle qui n'indique malheureusement pas la source utilisée pour la transcription ${ }^{49}$. Était-elle conservée alors en original dans les archives du chapitre ou copiée dans un cartulaire aujourd'hui perdu? En tout cas, le texte au style peu travaillé est sans nul doute très proche de loralité des débats. Il débute ainsi "Ce sont les paroles du jugement qu'à prononcé Adam à ceux présents devant l'évêque de Beauvais, Anseau ${ }^{50}$. Nous connaissons cet Adam. Il s'agit d'un casatus, c'est-à-dire un vassal de l'évêque, un membre de la petite noblesse qui vit dans la cité et que l'on peut suivre dans une poignée d'actes ${ }^{51}$. Adam joue dans plusieurs chartes un rôle d'expert juridique sans qu'il lui soit donné un titre particulier. S'il avait habité un peu plus au nord, on aurait certainement parlé de lui comme d'un échevin. Les casati forment à cette époque un groupe important de notables beauvaisiens qui souscrivent les actes, participent sans doute aux élections épiscopales, ont un privilège de sépulture dans la cathédrale ${ }^{52}$. Les casati siègent sans doute parfois dans la curia épiscopale ${ }^{53}$. La documentation est trop squelettique pour le prouver, mais on peut supposer que l'évêque composait sa curia soit de casati, soit de clercs, soit d'un mélange des deux suivant le degré ecclésiastique ou civil de laffaire à juger. Les casati constituent un groupe de chevaliers d'église dont les intérêts de classe devaient être assez proches de ceux des chanoines cathédraux. Adam est ainsi un juge-expert non professionnel, membre de la notabilité locale.

La notice est structurée de la manière suivante. Les chanoines avancent qu'ils ont un privilège qui interdit les planches, les poteaux et les terrassements à côté de leur moulin. Les habitants du bourg répondent que Guy leur a accordé des coutumes (consuetudines) à cet

1096-1099 (R. SPRANDEL, Ivo von Chartres und seine Stellung in der Kirchengeschichte, Stuttgart 1962, p. 188-189; 196).

${ }^{49}$ "Iudicium Adami. Haec sunt verba iudicii quod protulit Adans ceteris qui aderant assentientibus in presentia Anselli Blevacensis episcopi. Canonici clamaverunt molendinum esse impeditum de tribus rebus, scilicet de palis, de plancis, de terramento. Responderunt burgenses quod istam consuetudinem habuerant de IV episcopis ante eum et ipse episcopus eis suas consuetudines concesserat. Ideo iudicamus pro recto quod episcopus cuius est aque consuetudo et nullus eo contradicit debet facere deliberare cursum aque de predictis impedimentis ita quod molendino nihil noceat et homines ibi habeant ipsa necessaria que cursui aque non noceant et episcopus debet iubere ut bene fiant " (A. Loisel, Mémoires des pays ... de Beauvais et du Beauvaisis, Paris 1617, p. 266-267).

${ }^{50}$ Il est délicat de dire si le jugement a eu lieu à la curia épiscopale. En tout cas, dans les années 1114-1118, les procès ont lieu devant le châtelain de Beauvais (cf. infra). Il semble qu'il s'agisse des mêmes juges occasionnels qui agissent devant l'évêque ou devant le châtelain (O. GuYotjEAnnin, Episcopus et comes (cf. n. 19), p. 114).

${ }^{51}$ Ibid., p. 108.

${ }^{52} \mathrm{Ibid}$., p. 97 et passim. Le terme de casatus est utilisé à Beauvais entre 1078 et 1130.

${ }^{53}$ C'est ce que pense Labande en observant que cette obligation d'assister aux plaids épiscopaux est mentionnée pour les francs-hommes, une qualification qui apparaît plus tardivement et qui prend le relais des casati (L.-H. LaBANDE, Histoire de Beauvais (cf. n. 19), p. 140-142). 
égard et qu'elles n'ont pas été remises en cause par les évêques suivants. Adam prend alors la parole et juge en rectitude (pro recto) que la coutume (consuetudo) de leau appartient à lévêque et que c'est à l'évêque de faire en sorte que le flux d'eau qui accède au moulin ne soit pas perturbé par des obstacles. Les habitants du bourg peuvent aménager la rivière tant que cela ne gêne pas les autres utilisateurs.

Nous sommes ici dans une ambiance toute différente d'un plaid, figure classique de l'histoire judiciaire depuis le haut Moyen Âge ${ }^{54}$. Prenons un exemple local antérieur de trente ans. En 1069, lévêque Guy et d'autres évêques de diocèses voisins célèbrent la dédicace de Saint-Quentin. À cette occasion, écoutant la clamor des chanoines sur leur trésorier Garin qui avait accaparé une partie de la dîme, Guy contraint Garin à venir s'expliquer. Au plaid, accablé par tant de symboles spirituels (la dédicace, les évêques, les reliques), Garin s'effondre, reconnaît son péché et vient à satisfaction ${ }^{55}$. C'est ce rassemblement exceptionnel, cet aréopage mitré, qui provoque l'exercice de la justice. Les juges ne sont pas des experts mais des puissants. En 1099, Adam n'est plus larbitre passif de laffrontement des parties; il dit la règle de droit. Comme la bien montré Robert Jacob cette transformation du métier de juge est un tournant majeur du xII ${ }^{\mathrm{e}}$ siècle $^{56}$. La notice du jugement d'Adam est ainsi un témoignage de la sortie du monde du plaid et du rôle moteur que jouent dans la France du Nord les échevins - terme pris ici dans une acception lâche — dans la juridicisation de la société. Le deuxième aspect juridique saillant est la place accordée à la coutume.

Notre deuxième source sur le conflit à Beauvais est une autre lettre envoyée par Yves de Chartres au doyen du chapitre Hugues pour appuyer sa position et lui proposer un argumentaire juridique ${ }^{57}$. Par son érudition des sources canoniques, Yves offre un petit exposé doctrinal sur la coutume ${ }^{58}$. Il ne rejette pas la proposition des chanoines d'appuyer leur position sur le privilège mais il ne s'étend pas. La lettre quelque peu magistrale d'Yves ne doit pas occulter qu'il est en fait un insider du conflit et qu'il vise avant tout à s'attirer les bonnes grâces d'Hugues et ainsi concilier le chapitre cathédral et son alma mater. Ancien prévôt de Saint-Quentin, Yves a été le témoin de plusieurs chartes de lévêque Guy. Il connaît bien le terrain local et devait se douter que les 'antiques' parchemins sortis des coffres de la cathédrale sentaient l'encre fraîche. D'où son insistance sur la coutume.

Largumentation juridique d'Yves tourne autour de la prescription trentenaire ${ }^{59}$. Il oppose cette bonne règle frappée du sceau de la tradition canonique à la norme ridicule et sans fondement d'une prescription d'un an que les habitants de la cité (lire ici à mon avis 'du

\footnotetext{
${ }^{54} \mathrm{La}$ littérature sur le sujet est aujourd'hui très abondante, voir en dernier lieu : Fr. BougARD, «Écrire le procès ;

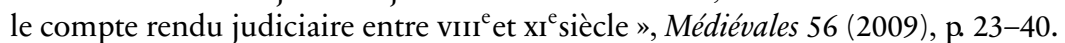

${ }^{55}$ L.-H. LABAnde, Histoire de Beauvais (cf. n. 19), p. 260-61.

${ }^{56} \mathrm{R}$. Јасов, «Le serment des juges ou linvention de la conscience judiciaire (xII européen) », in : Le Serment, éd. R. Verdier, Paris 1991, t. 1, p. 439-57.

${ }^{57}$ Yves de Chartres, Epist. 87 (PL 162, col. 98-99).

${ }^{58}$ Sur la coutume chez Yves de Chartres voir A. Lefrebvre-Teillard, «Custom and Law ", in : Proceedings of the Tenth International Congress of Medieval Canon Law, éd. K. Pennington; S. Chodorow/K. H. Kendall, Vatican 2001, p. 753-65 avec une analyse de la lettre 87.

${ }^{59}$ Sur la place et les fortunes de la prescription acquisitive dans Pordre juridique médiéval et ses rapports avec la coutume voir limportant mémoire de E. Conte, «Vetustas. Prescrizione acquisitiva e possesso dei diritti nel Medioevo ", in : Uso, tempo, posesso dei diritti. Una ricerca storica e di diritto privato, éd. E. Conte ; V. ManNino/P. Maria Vecchi, Torino 1999, p. 49-128.
} 
bourg') se seraient vue concéder par l'évêque Guy. Ils considéreraient cette prescription annuelle comme une coutume de la ville et auraient même fait serment de défendre lapplication de cette norme. Yves, sans difficulté, assène qu'une coutume qui contredit une loi ecclésiastique, la prescription trentenaire, n'a aucune valeur et doit être abolie. On le voit, la lettre d'Yves et la sentence d'Adam ne concordent pas totalement. On ne sait pas exactement quelle était la coutume dont se sont prévalus les bourgeois. S'agissait-il du droit d'aménager les rivières, d'une règle de prescription d'un an ou d'une combinaison de ces arguments?

\section{Penser Le MOMent COUTUMier De LA FIN DU XI ${ }^{\mathrm{E}}$ AU DÉBUt DU XII ${ }^{\mathrm{E}}$ SIÈCle}

Le conflit autour du moulin de Beauvais exemplifie le type de droit coutumier qui va nous intéresser. Récemment, certains historiens allemands du droit ont proposé de distinguer le Gewohnheitsrecht (droit coutumier) des Rechtsgewohnheiten (usages juridiques) ${ }^{60}$. Le Gewohnheitsrecht renverrait à la codification écrite des droits coutumiers et nécessiterait un ordre juridique hérité du modèle romano-canonique ; le paradigme en serait le Sachsenspiegel. Les Rechtsgewohnheiten désigneraient les voies du droit dans la société de l'oralité d'avant le $\mathrm{XII}^{\mathrm{e}}$ siècle où le droit reste encore enchâssé dans le social et le rituel, un concept qui n'est pas sans rappeler le prédroit cher à Louis Gernet ${ }^{61}$. Cependant, le conflit de Beauvais ne rentre pas dans cette distinction. Si la lettre d'Yves de Chartres est un maillon de la généalogie de la coutume romano-canonique, le jugement d'Adam n'est pas une règle du jeu acceptée par tous qu'il faudrait affirmer à nouveau pour obtenir justice ${ }^{62}$. Au contraire, il y a un conflit sur la norme objective à appliquer. Pour les bourgeois de Beauvais, les consuetudines auxquelles ils font référence ne sont pas du droit coutumier (Gewohnheitsrecht) dans le sens qu'il y aurait un corpus de règles juridiques orales connues de tous. Elles ne témoignent pas non plus de Pexistence depuis le milieu du xi ${ }^{\mathrm{e}}$ siècle d'une commune dans le sens d'un organe politique d'autogestion stable ${ }^{63}$. Guy a certainement accordé une charte de franchise aujourd'hui perdue au bourg qui leur accordait des consuetudines fiscales. Cependant, les bourgeois se pensent en sujets de droit et affirme leur identité juridique en avançant une norme, la prescription d'un an, qui est typique d'une société urbaine de marchands et d'artisans et qui se pose explicitement en opposition avec le temps de l'́́glise. Leur attitude est ici proche des Minangkabau. La norme identitaire se veut disruptive. Loin de Pimage d'Épinal d'un droit

\footnotetext{
${ }^{60}$ G. Dilcher et al., Gewohnheitsrecht und Rechtsgewohnheiten im Mittelalter, Berlin 1992 et le compte rendu de K. Kröschell, "Besprechung von Gerhard Dilcher, Reiner Schulze, Gewohnheitsrecht und Rechtsgewohnbeiten in Mittelalter, Berlin 1992 ", Quaderni fiorentini per la storia del pensiero giuridico 23 (1994), p. 428-434. Voir aussi la critique du concept de Rechtsgewohnheiten par A. CoRDES, «Rechtsgewohnheiten in lübischen Gesellschaftverträgen », in : Rechtsbegriffe im Mittelalter, éd. A. Condes/B. Kannowski, Frankfurt am Main, $\mathrm{p}$ 29-41 et l'analyse de lusage de consuetudo au Haut Moyen Âge par M. LupoI, The Origins of the European Legal Order, Cambridge 2000, p. 405-413 qui note que dans de nombreux textes, la coutume est synonyme de la loi ; le terme prend une signification autonome principalement dans son sens de taxes. La coutume n'est pas vue comme quelque chose d'oral et elle est rarement placée en dessous de la loi.

${ }^{61}$ L. Gernet, Anthropologie de la Grèce antique, Paris 1995.

${ }^{62}$ Sur cette idée de règle du jeu voir G. Althoff, Spielregeln der Politik im Mittelalter: Kommunikation in Frieden und Fehde, Darmstadt 1997.

${ }^{63}$ Position soutenue par L.-H. LABANDE, Histoire de Beauvais (cf. n. 19), p. 56-58 suivi par A. VermeEsch, Essai sur les origines et la signification de la commune dans le nord de la France : XI et XII' siècles, Heule 1966, p. 103-105.
} 
marchand orienté vers linformalité, la rapidité, le recours à larbitrage, le droit urbain naissant du XII ${ }^{\mathrm{e}}$ siècle témoigne en premier lieu du désir citadin de conquérir la violence légale. Obtenir la reconnaissance de la prescription annuelle comme coutume locale c'est légitimer lusucapion sauvage comme forme de violence collective rituelle.

Adam se place, lui, dans le mode de raisonnement savant de la règle de droit. Pour chaque rivière, il y a une coutume de l'eau. À Beauvais, celle-ci appartient à lévêque. Donc, il convient à ce dernier de faire appliquer la règle ${ }^{64}$. La solution pratique retenue est assez conforme aux pratiques médiévales de gestion des droits des eaux : la régulation par une autorité supérieure est la norme, la gestion communautaire par les stakeholders, de type poldermodel, lexception ${ }^{65}$. Qu'Adam applique les critères de rectitude ou de rationalité à la coutume n’a rien de spécifiquement étonnant si on reprend les leçons indonésiennes. Les élites locales sont conscientes que la coutume ne peut être un monument immobile. Au contraire, il ne peut survivre que s'il s'accorde avec le droit islamique et le droit d'origine européenne tout en conservant son identité. L'importation de concepts ou de modes de raisonnement externes est ainsi perçue comme un moyen de régénérer Padat.

L'outil conceptuel Gewohnheitsrecht / Rechtsgewohnheiten semble finalement peu utile pour comprendre le cas de Beauvais et plus largement sur ce que lon pourrait nommer le premier moment coutumier de 1050 à 1150 . Ce que lon nommera coutumes correspond à toutes ces normes explicitement attachées à des communautés définies. Ce lien organique entre norme et mouvement communautaire était du reste parfaitement analysé par certains textes médiévaux. En tête de son Cedrus, le maître de dictamen Boncompagno da Signa dresse une typologie des statuts en plaçant sur la même échelle le statut général — la loi impériale les statuts urbains mais aussi les statuts des compagnies de jeunesse et des copropriétaires de tours $^{66}$. Cette place forte donnée à Passociation libre comme récipiendaire de coutume est une différence majeure avec l'adat. Au Minangkabau, la coutume est fondamentalement liée à la famille et au clan; alors qu'au Moyen Âge central, elle s'accroche à une communauté artificielle : monastère, village, cité, confrérie...

On a voulu parfois distinguer coutumes subjectives (les vieilles coutumes fiscales) et coutumes objectives (règles de droit privé) ${ }^{67}$. Là encore, cette typologie me semble plus trompeuse qu'utile. Dans lordre juridique actuel, les droits subjectifs sont seconds par rapport

${ }^{64}$ La solution d'Adam est ainsi similaire à celle que proposent plus tardivement les romanistes lorsqu'ils traitent de la question scolaire suivante : que peut faire le propriétaire d'un moulin si la marche de son moulin est entravée par la construction d'un nouveau moulin en amont du premier. Il faut alors attaquer en justice le propriétaire du deuxième édifice en s'appuyant sur D. 43,13 : ne quid in flumine publico fiat, quo aliter fluat, quam in priori estate fluxerit. Voir N. SARTI, Inter vicinos praesumitur aemulatio. Le dinamiche dei rapporti di vicinato nell'esperianza del diritto commune, Milano 2003, p. 133-137.

${ }^{65}$ Voir ainsi les exemples donnés par G. CHIODI, «Conflitti per Puso delle acque nella Milano del XII secolo ", in : L'acqua nei secoli altomedievali, (Settimane di studio, 55), Spoleto 2008, p. 505-582 à comparer avec M. van TieLhof, "Op zoek naar het poldermodel in de waterstaatsgeschiedenis », Tijdschrift voor Geschiedenis 122 (2009), p. 148-161. À la fin du Moyen Âge, l'évêque de Beauvais a son sergent des eaux qui organise la gestion de la rivière et des canaux et fait la police (cf., supra, n. 20).

${ }^{66}$ Boncompagno da Signa, Cedrus 3 (http://scrineum. unipv.it/wight/cedrus.htm) avec cette définition : "Statutum est arbitraria mundi norma, que a uulgari hominum consuetudine procedit».

${ }^{67}$ Ainsi, M. AschierI, «Ancora tra consuetudini e statuti : prime esperienze (secoli X - XII) e precisazioni concettuali », in : Pensiero e sperimentazioni istituzionali nella Societas Christiana (1046-1250), éd. G. Andenna, Milano 2007, p. 167-198. 
aux droits objectifs; ils supposent que la norme objective détermine le concept de sujet de droit. Or, dans les textes les plus anciens, lidée de sujet de droit paraît totalement absente. C'est paradoxalement dans les coutumes les plus 'objectives', comme les privilèges judiciaires, que l'on trouve le plus de subjectivité.

L'outil analytique le plus fécond me semble, en dernier lieu, celui proposé par Anselme Davril pour classer les coutumiers monastiques. Le père Davril part de lanalyse de trois coutumiers clunisiens : celui d'Ulrich (ca. 1080), celui de Bernard (ca. 1085) et celui de Pierre le Vénérable (1146/47) et propose de distinguer le descriptif, le directif et le normatif ${ }^{68}$. Ulrich est descriptif puisque son ouvrage est une description des usages à Cluny pour son abbé Guillaume d'Hirsau ( «J'ai vu à Cluny que l'on a coutume de célébrer la liturgie de telle ou telle façon »). Bernard est directif car il souhaite fixer la coutume. Il est un membre éminent de la communauté qui se pose comme gardien et expert de la coutume clunisienne. Son texte n'a néanmoins pas la valeur normative des Statuts de Pierre le Vénérable qui, en tant qu'abbé, avait un pouvoir - théorique - d'imposer de nouvelles normes. À cette triade, je rajouterai un quatrième élément, celui de coutume identitaire dans le sens d'une coutume revendiquée comme propre à une communauté. Les Statuts de Pierre le Vénérable sont aussi un coutumier identaire car, écrits dans une ambiance de compétition avec les cisterciens, Pierre explicite pour chaque coutume sa ratio : "Si nous mangeons ainsi c'est parce que ... ». La coutume descriptive répondrait ainsi au principe de témoignage, la directive à un principe de vérité, la normative à un principe d'autorité et lidentitaire à un principe de rationalité. La coutume descriptive est mise par écrit pour les étrangers que lon cherche à instruire; la coutume directive et normative agissent dans la communauté avec un objectif réformateur : "Voici la coutume à appliquer car elle est vraie ou rationnelle.» La coutume identaire serait plus institutionnalisante dans le sens qu'elle constituerait l'un des marqueurs de l'identité de Pinstitution ou du groupe.

Le cas de Beauvais au début du XII ${ }^{\mathrm{e}}$ siècle permet de tester la pertinence de cette typologie. La coutume de leau avancée par Adam serait ainsi directive en tant qu'elle correspondrait à lidentification par un expert de la règle juste (mise en œuvre dans la société ou du moins dans la sanior pars) à appliquer. Un autre exemple provient du cartulaire de Saint-Quentin de Beauvais encore largement inédit. On y trouve plusieurs notices de plaids des premières décennies du XII ${ }^{\mathrm{e}}$ siècle dont l'étude apporterait sans doute des renseignements intéressants sur l'exercice de la justice à Beauvais à cette époque. Un fragment édité par Yvonne Bongert donne déjà un aperçu intéressant et offre la mention la plus ancienne de lexpression "la coutume de Beauvais ». En 1116, un certain Hugues de Ferrières entame une procédure judiciaire contre le monastère pour contester la possession de la moitié d'une maison. Hugues

\footnotetext{
${ }^{68}$ A. Davril, "Coutumiers directifs et coutumiers descriptifs d'Ulrich à Bernard de Cluny ", in : Du coeur de la nuit à la fin du jour. Les coutumes clunisiennes au Moyen Âge, éd. I. Cochelin/S. Boyton, Turnhout 2005, p. 23-28; sur ces textes, voir I. Cochelin, «Évolution des coutumiers monastiques dessinée à partir de létude de Bernard », in : Du cour de la nuit à la fin du jour. Les coutumes clunisiennes au Moyen Âge, éd. I. Cochelin/ S. Boyton, Turnhout 2005, p. 29-66 et plus largement lensemble de louvrage qu'elle codirige avec Susan Boynton. G. Melville, "Action, Text, and Validity : On Re-examining Cluny's Consuetudines and Statutes », in : Du cœur de la nuit à la fin du jour. Les coutumes clunisiennes au Moyen Âge, éd. I. Cochelin/S. Boyton, Turnhout 2005, p. 67-83 propose une typologie légèrement différente qui insiste sur le passage à l'écrit. Dans le cas de figure de Cluny, micro-société où l'écrit est très présent depuis le xi siècle, le modèle semble moins pertinent.
} 
affirme que cette moitié a été donnée à sa mère en dot et il en demande la restitution. L'abbé Raoul répond que cette maison est dans le patrimoine de labbaye depuis Yves de Chartres. Le châtelain Eudes qui préside le plaid annonce alors que le jugement doit être donné selon « la justice et la coutume de Beauvais (justiciam et consuetudinem Belvaci) ». Les juges déclarent alors que la coutume veut que ce soit la mère qui présente elle-même la clamor et on ajourne les débats. Le jour dit, à lassemblée judiciaire, la mère est présente mais refuse de parler. Son fils dit alors que c'est lui qui va présenter la clamor. Mais, le châtelain Eudes dit qu'il ne veut pas séparer justice et coutume (noluit separare justiciam et consuetudinem) et Saint-Quentin gagne ainsi le procès ${ }^{69}$. On peut se demander pourquoi la mère refuse de se défendre. Soit elle n’approuvait pas l'action de son fils soit elle était confrontée à un confit de coutume, entre les pratiques sociales qui veulent que ce soit l'homme qui gère le patrimoine familial et la coutume d'inspiration canonique, sans doute nouvelle, qui veut que laction en justice soit un droit qui traverse les sexes et les classes sociales ${ }^{70}$. Le casatus Adam est à nouveau présent parmi les juges du plaid et on peut supposer qu'il a joué un rôle moteur dans l'expression de cette coutume de Beauvais. En effet, si la procédure, celle du plaid, est plus traditionnelle que le jugement sur le moulin, on y retrouve malgré tout la marque de cette autonomisation du champ judiciaire. L'institution du châtelain apparaît dans le dernier quart du xi ${ }^{\mathrm{e}}$ siècle. Vassal de l'évêque, doté de fonctions militaires et policières difficiles à déterminer, il voit son statut social s'améliorer au cours des années pour intégrer le groupe des casati. Lascension d'Eudes II qui préside le plaid de 1116 sera stoppée par Louis VI qui bloquera ses exactions (exactiones) et ses coutumes (consuetudines) ${ }^{71}$. Ses revenus qu'il tire du minage, mesurage des grains, sont bornés. Il ne peut avoir un prévôt qui exerce sa justice dans toute la ville mais il peut rendre justice (justitia) à ceux qui portent leur clamor devant lui ou sa femme soit dans sa maison, soit dans le plaidoir ${ }^{72}$. La mention du plaidoir (placiterium) dans lacte de Louis VI est intrigante. Le mot semble très rare à cette époque ${ }^{73}$. On comprend dès lors mieux le langage d'Eudes II lors du plaid de 1116. Il existe ainsi un lieu dans Beauvais spécifiquement dédié à lexercice de la justice dont on aimerait connaître la localisation. Le plaidoir ne se confondait pas en tout cas avec le chastel, la place du marché ou laître de Saint-Étienne. Comme le remarque Luc Devliegher, Galbert, dans son célèbre récit du meurtre de Charles le Bon, indique qu'un grand nombre d'évènements collectifs, politiques ou judiciaires à Bruges se déroulaient au Sablon ('t Zand), un espace qu'il nomme parfois le champ coutumier (agrum consuetum) situé juste à la sortie du suburbium sur la route vers Ypres. Il existait pourtant à Pintérieur de la ville une vaste place du marché tout à fait adaptée aux réunions publiques ${ }^{74}$. C'est au Sablon que Louis VI et Guillaume Cliton lors de leur entrée à Bruges le 6 avril 1127 confirment en public les privilèges de Saint-Donatien et accordent aux bourgeois le droit de

\footnotetext{
${ }^{69}$ Y. Bongert, Recherches sur les cours lä̈ques du Xe au XII siècle, Paris 1949, p. 310-11.

${ }^{70}$ Sur les différentes coutumes qui accordent aux serfs le droit d'ester en justice, cf. Ch. de Miramon, « Guillaume de Champeaux et la règle de droit des personnes " (cf. n. 17).

${ }^{71}$ O. Guyotjeannin, Episcopus et comes (cf. n. 19), p. 184-85.

${ }^{72}$ Actes de Louis VI (cf. n. 29), nº 94.

${ }^{73}$ Selon les dictionnaires, à partir du xin ${ }^{\mathrm{e}}$ siècle, le plaidoir est la salle (ou le lieu) d'un tribunal municipal. Pour les époques antérieures, une première enquête dans les dictionnaires et bases de données n'a pas révélé d'autres occurrences du mot.

${ }^{74}$ L. Devliegher, "Galbert et la topographie de Bruges », in : Galbert de Bruges. Le Meurtre de Charles le Bon, éd. R. C. Van Caenegem et al., Antwerpen 1978, p. 255-256.
} 
faire évoluer leurs lois coutumières en les adaptant aux circonstances de temps et de lieu ${ }^{75}$. On peut analyser cet épisode comme un jalon dans la préhistoire du rituel politique de la joyeuse entrée ${ }^{76}$. Mais il est aussi important de noter la bilocalisation du droit soit replié dans la maisonnée - curia royale, épiscopale, féodale ou celle du châtelain de Beauvais - ou placé dans un lieu liminaire comme le plaidoir où le droit est perçu comme représentation vis-à-vis de l'extérieur. On comprend mieux alors la formule d'Eudes en 1116. S'il ne veut pas séparer la justice et la coutume, c'est qu'il exprime publiquement que sa justice obéit à une coutume de Beauvais dont il n'est pas maître. Dans la forme classique du plaid, dans une pure tradition cicéronienne, c'est aux parties et à leurs avocats de proposer aux puissants qui dirigent la procédure la norme à appliquer. Ici, ce rôle est dévolu au groupe des juges des plaids d'Eudes qui comme le remarque Olivier Guyotjeannin est similaire à celui que lon retrouve dans la curia de l'évêque ${ }^{77}$. Lors du plaid de 1116, la coutume de Beauvais ne sourd pas de la foule ni de la bouche des parties mais elle est fixée par une sanior pars de notables-juges.

En fin de compte, les marqueurs que l'on assigne traditionnellement à la coutume : oralité, ancestralité, subsidiarité ont ici peu d'importance. Dans les exemples que nous avons analysés, les traits saillants sont le caractère identitaire, la capacité à être incarné par des sujets de droit, la rationalité de la règle de droit. Ce portrait est sans doute réducteur car la coutume est un carrefour des consciences du droit. Tout comme pour la charte de Saint-Omer étudié dans larticle précédent, les consciences du droit ne sont pas toujours compatibles entre elles et apparaissent dans la documentation souvent plus juxtaposées que synthétisées. Ainsi, les tentatives de légalisation de la violence légale s'opposent à laffirmation d'une coutume rationnelle appuyée par la professionnalisation de la justice. Dans le complexe coutumier, c'est cette norme directive et identitaire qui nous intéresse car elle est le lieu paradoxal de réception sinon de textes savants du moins d'idées issues du droit canonique.

$\mathrm{Si}$ on suit les lignes de ce portrait, il semblerait que cette coutume directive et identitaire se dissout dans une catégorie très générique de norme. Pourquoi alors, le jugement d'Adam utilise le terme de consuetudo et pas un autre : regula, lex ou ius? Il ne s'agit cependant pas d'un simple hasard documentaire. Il existe une théorisation savante de la coutume contemporaine au jugement d'Adam dans lequel on retrouve cette idée coutumière qui s'écarte de lépure du Gewohnheitsrecht romano-canonique.

\section{Maingaud, Guillaume et le Droit Coutumier}

Le parcours le long du chemin sinueux de la naissance du droit coutumier aboutit à un lieu que nous avons exploré dans notre article du précédent volume : le monde communal de la France du Nord. Nous avons vu que les commentaires sur le De Inventione enseignés à Laon à la fin $\mathrm{xI}^{\mathrm{e}}$ siècle offraient des bribes de réflexion théorique sur la 'révolution communale. La

\footnotetext{
${ }^{75}$ Galbertus Brugensis, De multro, traditione et occisione Karoli comitis Flandriarum, éd. J. Rider, (Corpus Christianorum. Continuatio Mediaevalis, 131), Turnhout 1994, p. 104.

${ }^{76} \mathrm{La}$ bibliographie sur les cérémonies politiques urbaines à la fin du Moyen Âge et aux Temps modernes est aujourd'hui très abondante. Citons, entre autres, R. Descimon, « Le corps de ville et le système cérémoniel parisien au début de lâge moderne ", in : Statuts individuels, statuts corporatifs et statuts judiciaires dans les villes européennes (Moyen Age et Temps modernes), éd. M. Boone/M. PraK, Louvain 1996, p. 73-128.

${ }^{77}$ O. Guyotjeannin, Episcopus et comes (cf. n. 19), 114, n. 210.
} 
lecture de ces textes peut-elle aussi nous aider à mieux comprendre le moment coutumier du tournant du XI ${ }^{\mathrm{e}}$ et $\mathrm{XII}^{\mathrm{e}}$ siècle.

Nous conservons deux jeux de commentaires sur la rhétorique latine classique, le $D e I n$ ventione et la Rhétorique à Herennius (que le Moyen Âge attribuait aussi à Cicéron). Ces œuvres proviennent de l'école de Laon et sont contemporaines du début de l'enseignement d'Anselme de Laon. La première paire de commentaires est le produit du travail de maître Manegaldus, ici nommé Maingaud, et que certains - ce n'est pas mon opinion — identifient à Manegold de Lautenbach. La deuxième paire de commentaires reflète Penseignement de Guillaume de Champeaux. Son commentaire In Primis sur le De Inventione date des années 1095-1100. Celui de Maingaud est difficile à dater sur critères internes mais il est antérieur à Guillaume $^{78}$.

L'utilisation d'un commentaire médiéval d'une œuvre classique comme source historique est un exercice délicat. Maingaud et Guillaume cherchent avant tout à expliquer le texte cicéronien en tant que tel et pas à commenter la société qui les entoure. Les gloses sont majoritairement de nature antiquaire. Il s'agit d'expliquer tel mot compliqué, telle institution antique ou telle allusion que l'on trouve le texte. La plupart des exemples donnés sont volontairement décontextualisés et puisent dans un petit stock de références communes au monde romain : Caton et Carthage, Verrès, les Gracques, etc. Cependant, parfois, par de petits écarts, Maingaud et surtout Guillaume de Champeaux laissent apparaître autre chose que le commentaire scolaire.

Lun des aspects novateurs de ces commentaires est la place nouvelle accordée au droit. Certes, le De Inventione est essentiellement un manuel d'éloquence judiciaire. Néanmoins, si Porateur cicéronien est en premier lieu un avocat efficace c'est qu'il vise plus haut. La réussite de lorateur c'est de passer des succès de prétoire au couronnement d'homme politique. Cette figure romaine ne faisait pas beaucoup de sens dans la société de la fin du xi ${ }^{\mathrm{e}}$ siècle. Si Guillaume conserve à certains endroits de son commentaire cet orateur 'historique', à d'autres, il lui substitue un nouveau personnage, le sage (sapiens). Le sage est l'homme providentiel qui a fondé la communauté humaine et la placée sous le droit ${ }^{79}$.

On pourrait à première vue penser que ce sage est une réélaboration médiévale du jurisconsulte de la République romaine ${ }^{80}$. Il n'en est rien. Maingaud dans son commentaire du passage où Cicéron mentionne le jurisconsulte se contente de reformuler Marius Victorinus

\footnotetext{
${ }^{78}$ Je remercie vivement John Ward qui m’a communiqué son édition de travail du commentaire de Guillaume de Champeaux; ce qui m’a permis de lire l'ensemble du texte et d'améliorer mes propres transcriptions fragmentaires. Sur les commentaires sur le De Inventione et la Rhétorique à Herennius de la fin du XI ${ }^{\mathrm{e}}$ siècle et du XII ${ }^{\mathrm{e}}$ siècle voir J. O. WARD, «The Medieval and Early Renaissance Study of Cicero's De Inventione and the Rhetorica ad Herennium : Commentaries and Contexts ", in : The Rhetoric of Cicero in its Medieval and Early Renaissance Tradition, éd. V. Cox/J. O. WARD, Leiden 2006, p. 3-75 à la bibliographie duquel il faut ajouter K. M. Fredborg, "Petrus Helias's Summa on Cicero's De Inventione », Traditio 64 (2009), p. 139-182. Le commentaire sur la Rhétorique à Herennius Etsi cum Tullius ( $\mathrm{n}^{\circ} 8$ de la liste dressée par John Ward) est transmis dans les manuscrits avec In Primis et a donc été attribué aussi à Guillaume de Champeaux. Cependant, il présente des différences de style et de doctrine avec In Primis et je pense qu'il s'agit plutôt du travail d'un élève qui utilise le cours du maître assez librement.

${ }^{79} \mathrm{Ch}$. de Miramon, "Guillaume de Champeaux et la règle de droit des personnes » (cf. n. 17), p. 43.

${ }^{80}$ Sur les jurisconsultes de la fin de la République romaine, voir ainsi A. WATson, Law making in the later Roman Republic, Oxford 1974, chap. 7 et plus généralement Cl. Moatтi, La Raison de Rome. Naissance de l'esprit critique à la fin de la République (II ${ }^{e}-\mathrm{I}^{e r}$ siècle avant J.-C.), Paris 1997.
} 
et glose les juris consulti comme des juris periti ${ }^{81}$. Guillaume va s'écarter de la ligne définie par Marius Victorinus. Derrière les iuris periti, il voit des pères conciliaires, des prélats sages et savants qui collectivement sous lautorité du pontife formulent de nouvelles règles ou tranchent d'importants litiges ${ }^{82}$. Pour Guillaume, ces sages agissent pour la communauté en tant qu'expression la plus qualifiée de celle-ci. Guillaume n'emploie pas l'expression de sanior pars mais Pidée est présente. Donnons un seul exemple. Au paragraphe 47 du premier livre du De inventione, Cicéron définit le iudicatus, ce qui a été jugé. Il ne s'agit pas du concept juridique de la chose jugée mais d'une catégorie plus vague qui englobe ce que nous jugeons correct et que Cicéron divise de la manière suivante :

Ce qui est déjà jugé (iudicatum), c'est ce qui a été approuvé par lassentiment, lautorité, la sentence d'une ou de plusieurs personnes. On le divise en trois genres : sacré, général, confirmé. Est sacré ce qui a été jugé en vertu des lois par des personnes ayant prêté serment. Est général ce que tout le monde approuve et pratique. Exemple : se lever devant des gens plus âgés; avoir pitié des suppliants. Est confirmé un fait que des gens ont décidé, alors qu'on ne savait comment lapprécier, d'entériner de leur autorité, comme le peuple romain le fit pour la conduite de Gracchus le père, en Pélisant consul après sa censure, parce qu'il n'avait rien fait durant cette magistrature sans laccord de lautre censeur ${ }^{83}$.

Dans le commentaire In Primis, on trouve une première série de gloses qui paraphrasent cet attelage étrange et une deuxième explication qui recompose le concept en s'écartant quelque peu du texte. Ce qui a été jugé c'est ce qui a été confirmé par tous. Comme cet accord unanime est peu probable alors le iudicatus est ce qui a été décidé par les sages dans une opinion (sententia) écrite ou verbale. Ou, à défaut, le jugement donné par des juges ${ }^{84}$. Dans son introduction, Guillaume donne de la sagesse la définition suivante : « la sagesse est la connaissance de la vérité avec lintention de faire le bien ou de donner conseil ${ }^{85}$ ». À la différence de lora-

\footnotetext{
${ }^{81}$ Manegaldus, In De Inventione, 1, 11, 14 : «'Consulti iuris' : sunt idem qui et periti ab his enim merito consulimus. 'ius' qui habent scientiam iuris et quodammodo facilius est novum ius ex equitate et precedentis iuris informare » (Heidelberg, UB, Hs. 100, fol. 5v; Köln, Dombibl., ms. 197, fol. 12v) à comparer à Marius VictoRINus, Explanationes in Ciceronis Rhetoricam, ad eod. loc (Marius VICTORINus, Explanationes in Ciceronis Rhetoricam, éd. A. Ippolito, (Corpus Christianorum. Series Latina, 132), Turnhout 2006, p. 66). Pour Guillaume, les consulti iuris sont des juges (York, Minster Library, ms. XVI. M. 7, fol. 10r).

${ }^{82} \mathrm{Ch}$. de Miramon, "Guillaume de Champeaux et la règle de droit des personnes » (cf. n. 17), p. 43 et Idem, "Spiritualia et Temporalia - Naissance d'un couple », Zeitschrift der Savigny-Stiftung für Rechtsgeschichte. Kanonistische Abteilung 123/92 (2006), p. 224-287, p. 284.

${ }^{83}$ Cicéron, De l'invention, éd. établie et trad. par G. Achard, (Collection des Universités de France), Paris 1994, p. 101 mais voir la définition ultérieure du iudicatum plus juridique, infra n. 99.

${ }^{84}$ Willelmus Campellis, In De Inventione, 1, 30, 48 : «Iudicatum est illud quod est comprobatum assensione omnium. Differt a credibili quia credibile est cui statim credit auditor ; hoc comprobatum est, id est assensione confirmatum omnium, id est quia omnes dicunt creditur. Itaque differunt in approbato, aut si non omnium assensione approbatum est, saltem comprobatum est auctoritate sapientum, quod sapientes dederunt inde sentenciam uel uerbo, uel scripto, non tamen in iudicio; uel si hec duo non sunt, comprobatum est iudicio dato in causis, uel unius iudicis, uel multorum, et est diffinitio una facta sub diuisione. Differt sentencia a iudicio quia iudicum est quod a solis iudicibus datur " (York, Minster Library, ms. XVI. M. 7, fol. 20r). Et aussi : Willelmus Campellis, In De Inventione, 1, 28, 43 : «Differt sentencia a iudicio quia iudicum est quod a solis iudicibus datur; sentencia quod ab aliis, scilicet a tribunis qui non sunt iudices, et ab aliis » (York, Minster Library, ms. XVI. M. 7, fol. 19v).

${ }^{85}$ Willelmus Campellis, In De Inventione, Praef. : "Sapientia est cognitio ueritatis cum intencione boni uel consulendi » (York, Minster Library, ms. XVI. M. 7, fol. 2r).
} 
teur perçu comme individu, les sages comme les juges forment un groupe anonyme. Tous les deux jugent par sententia se rapprochant du travail des dialecticiens ou des maîtres. Cependant, contrairement aux juges, les sages participent du genre délibératif qui vise l'utilitas avant la vérité. Il y a ainsi dans le commentaire In Primis, esquissée plus que définie, une définition de lexpertise juridique à la fois comme travail sur les normes dans le but du bien commun mais aussi comme vérité scientifique. Il y a aussi un portrait du juriste qui differe du magister du Midi et de PItalie du Nord. Le juriste pour Guillaume est un membre de la sanior pars, échevin ou prélat, qui dit le droit avec ses confrères. Cette place première du collectif explique sans doute le rôle joué par la coutume et la loi populaire dans ces commentaires.

La lecture du De Inventione offrait un imaginaire de la fonction législative très différent de celui qui se dégagera du Corpus iuris civilis. Celui qui ne fait que feuilleter le Code remarque immédiatement limportance de lempereur, et en premier lieu de Justinien. Et, on le sait, la toute-puissance législative de lempereur eut une influence majeure sur la pensée juridique médiévale, en particulier sur le pontificalisme juridique. Au contraire, la lecture de Cicéron évoque une loi rare et solennelle qui émane de la volonté populaire. Commentant une brève allusion au mécanisme de création de la loi au paragraphe 134 du deuxième livre, Guillaume renvoie aux comices ${ }^{86}$. Maingaud et Guillaume ignorent le rôle de la magistrature dans lintroduction des lois devant cette institution. De même, Guillaume a une vision étriquée du préteur. Il pense que son édit ne concerne que les affaires militaires ${ }^{87}$. Le préteur de la République romaine ressemblerait à un châtelain urbain de la fin du $\mathrm{xI}^{\mathrm{e}}$ siècle. Les commentaires laonnois renvoient ainsi une image de Rome déformée dans le sens démocratique.

Cette place très forte accordée à lassemblée des citoyens se retrouve dans le traitement des sources du droit. On trouve, en effet, dans le De Inventione un court traité des sources du droit aux paragraphes 65 à 68 du deuxième livre. Cicéron explique que lorateur qui doit argumenter une controverse légale (negotialis), c'est-à-dire un point litigieux qui concerne la règle de droit à appliquer dans laffaire en procès, peut trouver du matériel (precepta) soit dans le droit issu de la nature, soit dans le droit issu de la coutume, soit dans le droit issu de la loi.

Le travail interprétatif médiéval consiste à passer du texte cicéronien à la distinction entre 'droit naturel', 'droit coutumier' et 'droit écrit'. Il faut bien comprendre que cette tripartition si naturelle dans la pensée juridique occidentale contemporaine n'existe pas dans le texte du $D e$ Inventione. Cicéron ne distingue pas des catégories juridiques autonomes, ni ne hiérarchise les sources. Il propose des trucs à l'orateur : la norme $\mathrm{X}$ qui est avantageuse à mon client est valide car elle est naturelle, car elle correspond à la coutume, car on peut la lire dans telle loi...

Comme la montré Franck Roumy, ce passage du De Inventione acquiert une signification nouvelle à partir du xi ${ }^{\mathrm{e}}$ siècle. Abbon de Fleury linsère ainsi dans sa collection canonique au chapitre traitant de la loi et de la coutume ${ }^{88}$. Cicéron devient une autorité sur les sources

\footnotetext{
${ }^{86}$ Willelmus Campellis, In De Inventione, 2, 45, 134 : «'sed apud populum, etc' a loco, a tempore, et quasi diceret, in foro ubi placita sunt non est corrigenda sed in Martio campo nec in hoc tempore sed in comiciis " (York, Minster Library, ms. XVI. M. 7, fol. 46v).

${ }^{87}$ Willelmus Campellis, In De Inventione, 2, 22, 67 : «'Et' sunt 'etiam eorum multo maxima pars illam quam pretores edicere consueverunt' : sicut claudere portas civitatis, vigiles per vicos mittere nocte, et alia que solent pretores iubere » (York, Minster Library, ms. XVI. M. 7, fol. 37r).

${ }^{88} \mathrm{Fr}$. Roumy, «Lex consuetudinaria, Jus consuetudinarium. Recherches sur la naissance du concept de droit coutu-
} 
du droit que les juristes médiévaux peuvent contraster avec ce qu'en dit Isidore de Séville. L'histoire textuelle des chapitres sur la loi et la coutume des Étymologies présente en effet des similitudes mais aussi des divergences avec le traité des sources du droit du De Inventione. Ces extraits de lencyclopédie d'Isidore circulent de manière autonome comme canons de collections ou incorporés dans des ouvrages juridiques didactiques ${ }^{89}$. Le statut d'autorité de ce passage du De Inventione explique que le commentaire des maîtres laonnois s'écarte de la simple explication de texte. Du reste, c'est dans ces commentaires que se voit clairement le passage de la terminologie cicéronienne - droit issu de la nature, droit issu de la coutume - à celle médiévale de droit naturel écrit et coutumier. Si lon replace ces occurrences dans le panorama dressé par Franck Roumy, Maingaud et Guillaume sont sans doute parmi les premiers à utiliser ces nouveaux termes ${ }^{90}$.

Tant lutilisation du terme droit (ius) que la distinction de différents types de droits est une nouveauté de la deuxième moitié du $\mathrm{xI}^{\mathrm{e}}$ siècle. Maurizio Lupoi montre que le concept de droit s'exprime depuis le Haut Moyen Âge jusqu'au xi ${ }^{\mathrm{e}}$ siècle soit par le mot 'loi' (lex) ou par le syntagme 'lois et coutumes' ${ }^{91}$. Ce trait de mentalité, qui voit la loi et la coutume comme de proches cousines, explique que Maingaud ne propose pas une tripartition droit naturel, droit écrit, droit coutumier mais une bipartition droit naturel, droit coutumier; ce dernier comportant tant le droit écrit, c'est-à-dire la loi, que le droit oral ${ }^{92}$. Plus en avant dans son commentaire, Maingaud propose même une autre division : il y a un seul droit, le droit coutumier qui se répartit entre droit naturel, droit des mores et droit légal ${ }^{93}$. Pour Maingaud, le terme de coutume a une double signification. Il désigne lensemble des normes en usage dans une société et aussi la part des normes qui sont uniquement orale ${ }^{94}$.

Le commentaire In Primis adopte une division différente des types de droit. Dans son explication du paragraphe 65 du deuxième livre, Guillaume distingue une tripartition droit naturel, droit coutumier, droit légal ${ }^{95}$. Mais, plus généralement, dans son commentaire, Guillaume

mier aux xI ${ }^{\mathrm{e}}$ et xII ${ }^{\mathrm{e}}$ siècles ", Revue historique de droit français et étranger 79 (2001), p. 257-291, p. 279-80.

${ }^{89}$ Fr. Roumy, «Lex consuetudinaria, Jus consuetudinarium » (cf. n. 88), p. 278.

${ }^{90}$ Ils sont contemporains de lemploi de la tripartition dans une lettre datée de 1084 et conservée dans les Regensburger rhetorischen Briefe (Ibid., p. 269).

${ }^{91}$ M. LupoI, The Origins of the European Legal Order (cf. n. 60), chap. 12.

${ }^{92}$ Manegaldus, In De Inventione, 2, 22, 65 : « Ius aliud naturale, aliud consuetudinarium. Consuetudinarium aliud scriptum id est lex, aliud non scriptum » (Heidelberg, UB, Hs. 100, fol. 22v).

${ }^{93}$ Manegaldus, In De Inventione, 2, 22, 65 : «Ius consuetudinarium aliud naturale quod magis per usum factum est, aliud quos ex vetustate in morem ductum est ut pactum, par, iudicatum, aliud legale » (Heidelberg, UB, Hs. 100, fol. 22v).

${ }^{94}$ Cicéron explique qu'il existe des coutumes dont la raison est évidente et d'autres dont lorigine est obscure. Maingaud propose pour cette catégorie de coutume obscure deux exemples intéressants : Manegaldus, In De Inventione, 2, 22, 65 : «Obscura ut hoc cur aliquis post exsectionem ungium eodem cultello aut forcipe secet $<$ se $>$ aut cur sponsi in nuptiis colaphi dentur ab amicis et multa id genus » (Heidelberg, UB, Hs. 100, fol. 22v). La première, si je comprends bien le latin ici corrompu, explique que celui qui se coupe les ongles doit s'entailler la main avec les ciseaux et la deuxième mentionne une gifle rituelle donnée aux époux lors de la noce. Je n'ai jamais rencontré ailleurs mentions de telles coutumes (E. HofFMANN-KraYer/H. BäChtold-STAüBLI (éd.), Handwörterbuch des deutschen Aberglaubens, Leipzig 1927-1942, t. 6, col. 1217-1218, s. v. 'Ohrfeige' mentionne sans références la gifle lors des noces). La gifle est un élément connu du rituel de la vassalité et de certaines cérémonies de donation.

${ }^{95}$ Willelmus Campellis, In De Inventione, 2, 22, 65 : « Nota quod ius aliud naturale, aliud consuetudinarium, aliud legale » (York, Minster Library, ms. XVI. M. 7, fol. 36v). 
adopte une bipartitation entre droit légal et droit coutumier ${ }^{96}$.

Cette négligence du droit naturel par Guillaume est révélatrice du désintérêt des commentateurs pour ce type de norme. Cicéron définit le droit issu de la nature comme un instinct, une vis innata, et il en offre un panorama très large : religion, sens du devoir envers sa patrie, sa famille et ses proches, gratitude, désir de vengeance, sens du respect et loyauté. Le commentaire de Maingaud reste proche de Cicéron. Il se borne à définir les différents termes du texte source. Seuls les exemples d'arguments utilisant le droit naturel proposés par Maingaud montrent une certaine originalité. Maingaud explique qu'un avocat pourrait avancer dans un procès : "Qu'il n'est pas étonnant que cet homme prenne soin de ses filles car les bêtes sauvages à qui la raison fait défaut s'occupent aussi de leur progéniture ». On retrouve ici lidée, absente tant du De Inventione que de la Rhétorique à Herennius, que l'homme partage avec Panimal le droit naturel et dont la plus célèbre expression se trouve dans le fragment d'Ulpien qui ouvre le Digeste ${ }^{97}$. Guillaume reprend la trame du commentaire de Maingaud mais est gêné par l'hétéroclite droit naturel cicéronien. Il en réduit drastiquement le champ en le limitant à la piété familiale (c'est le contenu du droit naturel selon la Rhétorique à Herennius II, 19) et à la religion ${ }^{98}$.

Ainsi, contrairement aux efforts postérieurs des juristes bolonais, le droit naturel intéresse peu les commentateurs laonnois. Le point axial de leur réflexion est la coutume dans son ambivalence, règle générique mais aussi norme spécifique orale. Cette ambiguïté, Maingaud et Guillaume la retrouvent dans le passage suivant de Cicéron :

On considère comme fondé sur la coutume le droit qui a été consacré par lancienneté, en raison du consentement général, sans sanction de la loi. Il comporte même des principes qui en raison de leur ancienneté ont pris une valeur juridique confirmée. Dans ce type de droit en effet ils abondent, mais se sont ceux que les préteurs ont eu Phabitude de faire figurer dans leurs édits qui sont de loin les plus nombreux. De plus, certains sont même devenus avec la coutume des catégories précises du droit. Par exemple, les conventions, l'équité, les précédents juridiques. Une convention est considérée comme tellement juste par les parties qui l'ont conclue qu'elle l'emporte sur le droit même; P'équité, elle, est juste pour tout le monde ; un précédent juridique est quelque chose qui a été déjà tranché par le jugement d'une ou plusieurs personnes ${ }^{99}$.

\footnotetext{
${ }^{96}$ Par exemple : Willelmus Campellis, In De Inventione, 1, 28, 43 : «Inde ut sic probet : uere tenenda est quia hoc lex precepit illa, uel usus hominum qui ita habent in consuetudine» (York, Minster Library, ms. XVI. M. 7, fol. 19r).

${ }^{97}$ Pour la fortune ultérieure de ce texte, voir R. Weigand, Die Naturrechtslehre der Legisten und Dekretisten von Irnerius bis Accursius und von Gratian bis Johannes Teutonicus, München 1967.

${ }^{98}$ Willelmus Campellis, In De Inventione, 2, 22, 65 : « Naturale ius est illud ius non quod affert opinio hominum nobis, sicut illa que in consuetudine sunt, sed quod 'affert quedam vis' ipsius iuris 'innata', id est naturalem vim habet in se quare tenendum se indicat, sicut colere deum, amare filium, etc. » (York, Minster Library, ms. XVI. M. 7, fol. 36v).

${ }^{99}$ Cicero, De Inventione, II, 67 : «Consuetudine autem ius esse putatur id quod voluntate omnium sine lege vetustas comprobarit. In ea autem quaedam sunt iura ipsa certa propter vetustatem. Quo in genere et alia sunt multa et eorum multo maxima pars quae praetores edicere consuerunt. Quaedam autem genera iuris iam certa consuetudine facta sunt; quod genus pactum, par, iudicatum. Pactum est quod inter quos convenit ita iustum putatur ut iure praestare dicatur; par, quod in omnes aequabile est; iudicatum, de quo iam ante sententia alicuius aut aliquorum constitutum est. Iam iura legitima ec legibus cognosci oportebit »(CICÉRON, De l'invention (cf. n. 83), p. 177).
} 
Les commentateurs vont essayer de clarifier lobscurité du texte en proposant des typologies de la coutume.

Maingaud explique d'abord que le droit coutumier est soit écrit, soit oral. Tout commence par une coutume qui est ensuite approuvée (adprobata) à cause de son utilité ou de son usage social. Cette approbation en fait du droit coutumier qui est ensuite fixé (firmatus) par sa mise à l'écrit sous forme de loi écrite ${ }^{100}$. Maingaud revient ensuite sur ce droit coutumier non écrit. Il existe une part très ancienne (vetustissima) qui reste orale car elle n'est pas codifiée et donc inapte au passage à Pécriture. Le reste du droit coutumier est plus récent et il est quasiment de la loi car déjà divisé sous forme de normes distinctes. Maingaud rattache cette pré-loi au préteur ${ }^{101}$. Le petit cœur du droit coutumier oral correspond à la mos isidorienne. En effet, Isidore de Séville explique « qu'entre la loi et les mores, la différence est que la loi est écrite tandis que le mos est une coutume approuvée par lancienneté ou encore une loi non écrite ${ }^{102} »$. Loralité renvoie à un passé lointain, préhistorique.

Finalement, Maingaud n'accorde aucune importance à la coutume. Maingaud dont le commentaire montre qu'il est un bon connaisseur pour son époque de la Rome classique connaît du reste la vaste étendue du droit romain savant. Ainsi, lorsqu'il est question de droit écrit, Maingaud explique que : "celui qui veut le connaître doit lire le Code, les Novelles, les Institutes et les autres livres de droit ${ }^{103}$ ». Cette courte notation est intéressante pour l'histoire complexe de la redécouverte des corpus justiniens traditionnellement perçue comme une affaire largement italienne. Le récent ouvrage de Charles Radding et Antonio Ciaralli accorde une place centrale (trop centrale diront d'aucuns) à l'école de droit lombard de Pavie

${ }^{100}$ Manegaldus, In De Inventione, 2, 22, 65 : « Deinde dividit consuetudinem quasi dicit : consuetudinarium queddam scriptum est queddam remansit in sola consuetudine et hoc est quod dicit. Postquam autem quedam adprobata id est consuetudinaria. Consuetudo enim a multis adprobatur vel laudatur quia sunt ipsa utilia aut ex consuetudine sola aut ex 'v[oluntate]' id est propter manifestam utilitatem videntur firmata esse legibus id est scripta » (Heidelberg, UB, Hs. 100, fol. 22v).

${ }^{101}$ Manegaldus, In De Inventione, 2, 22, 67 : «Consuetudinarium ius non scriptum aliud vetustissimum nec certis nominibus designatum aliud notis et certis nominibus distinctum. 'In ea' id est in ea parte iuris quammodo dixi sunt quedam 'iura iam certa propter vetustatem'. Et priusquam aliam partem ponat subdividit hanc : 'Quo in genere' id est in hoc genere vetustissimo certis nominibus non distincta sunt et alia multa que ad pretores non pertinetur et 'eorum' id est ex his est 'pars multo maxima' que apud 'pretores' tantum invenitur " (Heidelberg, UB, Hs. 100, fol. 22v).

${ }^{102}$ IsidDorus Hispalensis, Etymologiae, II, 10, 1 : «Inter legem autem et mores hoc interest, quod lex scripta est, mos vero est vetustate probata consuetudo, sive lex non scripta " (IsIdore DE SÉville, Étymologies Livre II. Rhétorique et dialectique, éd. P. K. Marshall, Paris 1983, p. 51). Je reprends la traduction de Fr. Roumy, «Lex consuetudinaria, Jus consuetudinarium » (cf. n. 88), p. 278.

${ }^{103}$ Manegaldus, In De Inventione, 2, 22, 68 : «Iam iura legitima quasi dicit qui hoc scire voluerit legat Codicem, Novellam, Institutam et alios legales libros » (Heidelberg, UB, Hs. 100, fol. 22v). La singulière désignation au singulier 'l'Institute', 'la Novelle' se retrouve aussi dans les citations du droit justinien présentes dans un opuscule de droit lombard de la première moitié du xi ${ }^{\mathrm{e}}$ siècle, les Quaestiones ac monita (sur ce texte Ch. M. Radding/A. Ciaralli, The Corpus iuris civilis in the Middle Ages. Manuscript and transmission from the sixth century to the juristic revival, Leiden 2007, p. 78-80) : "Quia sic praecipit lex Romana in libro qui vocatur Novella, quae egit Iustinianus imperator temporibus suis ... iuxta illud quod dicitur in lege Romana in libro qui vocatur Instituta » (MGH, Leges IV, 590 ; 593). Pour la désignation des Novelles (c'est-à-dire la collection de novelles que Pon dénomme Epitome Juliani) comme Novella, cf. aussi W. KaIser, Die Epitome Iuliani. Beiträge zum römischen Recht im frühen Mittelalter und zum byzantinischen Rechtsunterricht, Frankfurt am Main 2004, p. 706. 
dans lacclimatation du droit romain à l'Europe médiévale ${ }^{104}$. Le commentaire de Maingaud montre qu'un enseignant de l'Europe septentrionale des années 1080 a accès ou imagine que lon puisse avoir accès à un corpus de droit justinien comparable avec ce que lon trouve de lautre côté des Alpes à cette époque ${ }^{105}$. Cependant, même si Maingaud connaît lexistence des compilations justiniennes, il les a peu ou pas lues. Il ne semble pas qu'il y ait de citation explicite du droit romain justinien dans son commentaire sur le De inventione ${ }^{106}$. Maingaud mentionne aussi lexistence du droit lombard ${ }^{107}$. Là encore, tout indique que ses connaissances en la matière sont superficielles. Pourtant, même si Maingaud est un généraliste qui ne montre pas une technique juridique équivalente à ce que lon trouve dans le Liber papiensis, il participe au même univers mental que les juges pisans ${ }^{108}$. La norme est avant tout un corpus volumineux de lois anciennes, constitutions impériales, capitulaires carolingiens ou canons de collections canoniques. Certes, il faut prendre en compte l'usage (usus) qui fait que certains principes ne sont plus appliqués ou sont corrigés par des textes plus récents. Mais, en fin de compte, le ius est l'héritage écrit d'un passé législateur.

Lapproche du droit coutumier par Guillaume est radicalement différente. Il n'accorde aucune importance à la distinction entre droit oral et écrit. Il saute aussi la mention des compilations justiniennes ainsi que lallusion au droit lombard ${ }^{109}$. L'obscure distinction faite par Cicéron entre deux types de coutume doit se comprendre, selon lui, comme opposant les coutumes qui sont anciennes en soi et celles qui sont anciennes selon la coutume. Les coutumes anciennes en soi correspondent à un noyau universel et intemporel de règles juridiques dont le paradigme serait linterdiction du vol. Les autres coutumes renvoient au droit nouveau qui est dit coutumier car il résulte de métarègles anciennes que Guillaume énumère : les normes décidées par un accord commun doivent être respectées, de même les conventions, une fois scellées, doivent être tenues ${ }^{110}$. Guillaume retrouve dans le terme difficile à traduire de par, léquité son idée de droits nouveaux. Les jura nova doivent être équitables dans le sens qu'ils

${ }^{104}$ Ch. M. Radding/A. Ciaralli, The Corpus iuris civilis in the Middle Ages. Manuscript and transmission from the sixth century to the juristic revival (cf. n. 103) et le compte rendu critique de Wolfgang Mueller dans Speculum (2008), 83, p. 1026-1027.

${ }^{105}$ Cette connaissance par Maingaud du Corpus iuris civilis indique que les matériaux de droit romain inclus par Yves de Chartres dans la Collectio britannica ne proviennent pas nécessairement de bibliothèques italiennes.

${ }^{106}$ D’après une première lecture rapide du manuscrit. Un travail minutieux pour distinguer ce qui provient de Cicéron ou du savoir antique et ce qui pourrait être justinien reste évidemment à réaliser.

${ }^{107}$ Manegaldus, In De Inventione, 1, 8, 10 : «'Qua lege' : longobardica vel romana » (Heidelberg, UB, Hs. 100, fol. 4v; Köln, Dombibl., ms. 197, fol. 10v).

${ }^{108}$ G. Diurni, "Consuetudine e legge nella prassi longobardo-franca ", in : El Dret comú $i$ Catalunya - actes del VI Simposi Internacional, éd. A. J. Iglesia Ferreirós, Barcelona 1997, p. 67-100.

${ }^{109}$ Dans son souci d'actualisation du texte, Guillaume remplace lopposition entre droits romain et lombard par celle entre droits anglais et français. Willelmus Campellis, In De Inventione, 1, 8, 10 : "'Qua lege' : quia Anglicus nollet iudicari lege gallica " (York, Minster Library, ms. XVI. M. 7, fol. 8v).

${ }^{110}$ Willelmus Campellis, In De Inventione, 2, 22, 67 : «Consuetudine : Postquam tractavit de naturali iure vult agere de consuetudinario. Consuetudinarium aliud vetustum quod est in se vetustum, aliud vetustum propter consuetudinem. Vetustum in se est illud quod per maximum tempus iam repertum est, ut qui fecerit furtum puniatur. Vetustum propter consuetudinem est sicut quando iura nova faciunt homines. Ipsa nova sunt sed consuetudo eorum vetusta, scilicet, quod constituta communia violanda non sunt. Similiter est de pacto. Cotidie facimus nova pacta sed consuetudo pacti vetus est, scilicet, quod pactum teneatur postquam firmatum est » (York, Minster Library, ms. XVI. M. 7, fol. 36v). 
doivent être égaux entre les riches et les pauvres de la communauté ${ }^{111}$.

Là où Maingaud a les yeux tournés vers le passé, vers un processus coutumier déjà arrivé à son terme, Guillaume perçoit la vitalité du droit présent. Le monde est plein de droit et chaque fois que les hommes passent convention ou décident en commun une règle équitable, le chant de la coutume résonne à nouveau et linstitution initiale est revivifiée.

Pour Guillaume, lordre juridique est en croissance dynamique. Il existe un stock de droits déjà connus, ce qu'il nomme le droit explicite, et un droit neuf qui comporte tant les règles fixées par la méthode de la constitutio negotialis que les droits nouveaux coutumiers. Les règles issues de la constitutio negotialis correspondent à ce que lon nomme aujourd'hui la doctrine; elles sont déduites des normes explicites par les iuris periti de manière scientifique ${ }^{112}$. Les iura nova coutumiers se fondent tant sur le droit des communautés à se choisir les normes qui les régulent mais aussi sur lobligation pour ces normes émergentes de se plier à un ordre juridique commun dont les experts en droit sont les gardiens.

La théorie de Guillaume offre un éclairage sur le moment coutumier du début du xII ${ }^{\mathrm{e}}$ siècle, sur le jugement d'Adam, sur laffirmation du châtelain Eudes mais aussi sur les documents étudiés dans le précédent article.

Le moment coutumier accompagne la montée de nouvelles identités dans lesquelles on ne doit pas séparer le religieux, le bourgeois et le féodal. Le droit coutumier directif est aussi un outil politique par lequel les rois et évêques cherchent à établir de nouveaux actes de pouvoir quitte à légitimer la demande locale de droits et de privilèges. Il est enfin au service d'une professionnalisation de la fonction juridique visible tant pour les élites urbaines que cléricales. Programme plus que réalisation, son résultat est sans doute modeste. Mais, en ce début de $\mathrm{xII}^{\mathrm{e}}$ siècle, les consciences du droit sont certainement plus fragmentées qu'elles ne le seront à la fin du siècle quand le droit romano-canonique aura fourni une grammaire juridique européenne. Contrairement à la plupart des intellectuels de son époque, Guillaume de Champeaux a perçu de manière positive le bourdonnement du moment coutumier et il a exposé une sociologie du droit originale.

Guillaume de Champeaux s'est beaucoup intéressé au langage et on pourrait faire Phypothèse que son idée de la coutume nouvelle comme revivifiant linstitution normative originelle s'inspire du concept d'imposition selon lequel la signification d'un mot à été instituée à un moment, même si le mot peut prendre par la suite des sens dérivés et secondaires ${ }^{113}$. Boèce dans son Commentaire sur le Peri Hermeneias mettait en parallèle l'opposition entre les expressions vocales qui signifient par nature et celles qui signifient « par position » et lopposition

\footnotetext{
${ }^{111}$ Willelmus Campellis, In De Inventione, 2, 22, 68 :"'Par' est illud ius quod noviter inventum factum est, id est 'equabile inter omnes', id est quod omnes in iure illo et divites et pauperes coequantur » (York, Minster Library, ms. XVI. M. 7, fol. 37r).

${ }^{112}$ Willelmus Campellis, In De Inventione, 2, 21, 62 : « Negotialis : Est illa que in ipso habet negotio implicitam controversiam civilis iuris, quia quid inde tenendum sit nondum est in lege vel consuetudine explicitum, sed exculpitur novum ius, id est ex precedenti iure aut per simile aut per contrarium. Unde negotialis dicitur quasi negans otium, quia quod nondum inventum est magno cum labore tractatur et etiam semper ad futurum spectat» (York, Minster Library, ms. XVI. M. 7, fol. 36).

${ }^{113}$ I. Rosier-Сatach, La parole efficace. Signe, rituel, sacré, Paris 2004, p. 57-68.
} 
entre le droit naturel et le droit civil qui est aussi institué «par position » ${ }^{114}$. Cependant, le terme 'positif' ou positio est absent du commentaire In Primis. Guillaume lie Pinstitution à la coutume tant pour le droit que pour le langage ${ }^{115}$. Cependant, les lecteurs et les élèves de Guillaume vont abandonner son concept de coutume pour adopter le terme nouveau de droit positif. C'est ainsi le cas d'Abélard et de Thierry de Chartres ${ }^{116}$. Dans ses Collationes, Abélard - ce qui pour l'instant n'avait pas été remarqué - utilise le cours de son maître Guillaume $^{117}$. De même, Thierry de Chartres est influencé dans ses commentaires sur la rhétorique par In Primis ${ }^{118}$. Pourtant ladoption par ses deux auteurs du terme de droit positif est une critique implicite de la place donnée par Guillaume au droit coutumier. L'imposition du nom est arbitraire (ad placitum). C'est par convention que le son 'vache' signifie un animal à cornes mugissant. De même, le droit positif est arbitraire. Le philosophe qu'Abélard met en scène dans les Collationes définit le droit positif et oppose deux droits de la preuve : l'ordalie et le serment. Cependant, il n'explique pas quel est le meilleur système, le plus en phase avec le droit naturel. Le droit positif varie et dépend des habitudes locales. Dès lors, la coutume se détache de la volonté populaire et de sa sanior pars; elle devient un accident historique et géographique.

Cet abandon de la sociologie du droit de Guillaume a des raisons contextuelles — Pessoufflement du moment coutumier - mais aussi intellectuelles. Le schéma juridico-historique dominant dans les écoles du Nord de la France du début du XII ${ }^{\mathrm{e}}$ siècle n'est plus profane mais

\footnotetext{
${ }^{114}$ Boetius, Commentarii in librum Aristotelis Peri Ermeneias, éd. K. Meiser, Leipzig 1877-1880, t. 2, p. 41-42 et A. Boureau, «Droit naturel et abstraction judiciaire. Hypothèses sur la nature du droit médiéval », Annales HSC $57: 6$ (2002), p. 1463-1488, p. 1470.

${ }^{115}$ Willelmus Campellis, In De Inventione, 1, 11, 15 : « Littera sic legitur : 'Iuridicialis est in qua queritur natura 'equi' : id est illius institucionis qua equantur ciues, que habita secundum consuetudinem aliquando est iusta, aliquando iniusta (York, Minster Library, ms. XVI. M. 7, fol. 10r). À comparer au commentaire sur Porphyre du Ps-Raban, récemment attribué à Guillaume de Champeaux par Yukio Iwakama : «Ne ita videretur quod hoc nomen quod est 'genus' prius esset ei impositum in humani consuetudine sermonis » (Y. IwAKUMA, "Pseudo-Rabanus super Porphyrium ", Archives d'histoire doctrinale et littéraire du Moyen Âge 75 (2008), p. 43-196, p. 90). Lexpression humani consuetudine sermonis provient du commentaire de Boèce sur l'Isagogue (Boetius, In Isagogen Porphyri commentorum editio secunda, éd. S. BRANDT, Leipzig 1906, p. 177).

${ }^{116}$ Sur Pinvention du droit positif voir A. Boureau, "Droit naturel et abstraction judiciaire » (cf. n. 114) et J. Marenbon, "Abelard's Concept of Natural Law", in : Mensch und Natur im Mittelalter, éd. A. Speer/A. ZIMMermanN, Berlin/New York 1992, p. t. 2, t. 2.

${ }^{117}$ Comparez ainsi Petrus Abaelardus, Collationes, 2, $132:$ «Sunt qui partes iustitie ampliori numero non rerum sed nominum distinguentes, plerasque a nobis uno comprehensas vocabulo pluribus distingunt, et quod in toto conclusum est, per partes discernunt : pietatem scilicet erga parentes, amictiam — id est benivolentiam erga eos qui nos diligunt, ipsorum causa magis quam sperandi alicuius commodi, cum pari eorum erga nos voluntate - gratiam in remuneratione beneficiorum » (Petrus Abaelardus, Collationes, éd. J. Marenbon/ G. Orlandi, Oxford 2001, p. 144) et Willelmus Campellis, In De Inventione, 1, 11, 15 : « [De Inv. II. 161] 'Nature' : Prius de naturali iure agit, quod non est secundum opinionem hominum sed ex quadam naturali vi rationis procedit, quod dividit in quatuor partes, sicut in negotiali constitutione. 'Pietas' : Est que habetur in parentes et in patriam et in socios quibus debemus 'officium', id est humanitatem, et 'diligentem' cultum. 'Gratia' : Est in qua 'memoria amiciciarum et officiorum', id est, beneficiorum, et 'voluntas continetur' reddere singula singulis. 'Memoria alterius', id est, 'amiciciarum', ut amicorum semper recordemur, et 'voluntas' alterius 'remunerandi', ut si non habeamus facultatem saltem habeamus voluntatem remunerandi beneficia » (York, Minster Library, ms. XVI. M. 7, fol. 50r). Abélard explique qu'il a suivi le cours sur la rhétorique de Guillaume (Pierre Abélard, Historia Calamitatum, éd. J. Monfrin, Paris 1978, p. 65)

${ }^{118}$ Thierry de Chartres, The Latin rhetorical commentaries, éd. K. M. Fredborg, Toronto 1988, p. 12.
} 
biblique et distinguait le temps de la loi naturelle, le temps de la loi de l'Ancien Testament et le temps actuel de la loi du Nouveau Testament. Cela créait un nouvel objet juridique : le droit des juifs sous l'Ancienne Loi, droit perçu par les intellectuels chrétiens comme en partie obscur, arbitraire, irrationnel. Le rapport au droit passé changeait. Le droit de jadis n'est pas toujours vénérable; il est parfois dépassé ${ }^{119}$. Du reste, dans sa sentence théologique sur le droit naturel qui correspond sans doute à son enseignement à Châlons-en-Champagne entre 1113 et 1117, Guillaume a abandonné toute utilisation de la théorie du droit cicéronienne ${ }^{120}$. La théorie de Guillaume aura malgré tout une influence sur les générations suivantes de juristes et de commentateurs du De Inventione ${ }^{121}$. Pourtant, elle sera éclipsée par la réception massive du prologue d'Yves de Chartres puis par la théorie des normes issue des écoles de Bologne.

Il y a ainsi un hiatus qui témoigne de la fragilité de la coutume dans l'Occident médiéval. Dans le Minangkabau, la coutume est liée à des institutions - la famille, le clan — qui impriment avec force la reproduction sociale sur les individus. En Occident la coutume s'accroche à des communautés artificielles qui ont bien plus de mal à transformer la coutume en seconde nature. Le Minangkabau est aussi une région qui a peu connu de pouvoir individuel centralisé. Certes, le Sumatra pré-colonial n'est pas régi un communisme agraire. Il y a des penghulu plus puissants que d'autres, des leaders religieux charismatiques, le lignage royal n'est pas sans importance. Cependant, il n'y a rien d'équivalent à un pouvoir centralisé individuel qu'il soit épiscopal, royal, impérial ou pontifical. Ce n'est donc pas étonnant que le moment coutumier correspond à un affaiblissement tant des pouvoirs centralisés laïcs que religieux. Pourtant, comme en témoigne les innombrables conflits autour des élections ecclésiastiques ou les piteuses révolutions communales, la plupart des tentatives de pouvoir

${ }^{119}$ Sur la catégorie du droit dépassé (ceremonialia) pour les canonistes, je me permets de renvoyer à Ch. de $\mathrm{MI}^{-}$ RAMON, "Déconstruction et reconstruction du tabou de la femme menstruée (xII ${ }^{\mathrm{e}}$-xIII ${ }^{\mathrm{e}}$ siècle) », in : Kontinuitäten und Zäsuren in der Europäischen Rechtsgeschichte, Europäisches Forum Junger Rechtshistorikerinnen und Rechtshistoriker, München 22.-24. Juli 1998, éd. A. Thier; G. Pfeifer/P. Grzimek, Frankfurt am Main 1999, p. 79-107.

${ }^{120}$ O. LotTin, Psychologie et Morale aux XII et XIII siècles, Louvain / Gembloux 1942-1960, t. 5, n. 261. Sur la datation des sentences théologiques de Guillaume, cf. Ch. de Miramon, «Quatre notes biographiques sur Guillaume de Champeaux », in : Arts du langage et théologie aux confins des XI et XII siècle, éd. I. Rosier-Catach, Turnhout 2011, p. 45-82.

${ }^{121}$ Certaines idées des commentaires laonnois percolent dans les dicta de Gratien. Ainsi, on trouve un écho de la définition de la coutume par Maingaud dans D. 1, d. p. c. 5 : la coutume est la catégorie générale de la norme qui n'est pas du droit naturel et elle se divise en ius constitutionis et ius consuetudinis (voir aussi D. 8, d. a. c. 1 et d. p. c. 1). Gratien fait aussi le lien entre première cité et invention du ius consuetudinis (à comprendre ici comme la catégorie normative englobante qui comporte tant le droit des coutumes que celui des constitutions) dans D. 6, d. p. c. 3. Il remplace le mythe cicéronien du héros inventeur de la civilisation par le récit biblique. Caïn puis Nemrod sont les inventeurs de la ville. La ville et le droit coutumier sont associés à des personnages funestes. Plus tard, on retrouve un lien entre coutume et loi populaire chez Étienne de Tournai (STEPHANus Tornacensis, Summa Decreti, D. 1 c. 5, v' 'nec dicta', Étienne de Tournai, Summa Decreti, éd. J. F. Schulte, réimpr. anast. Aaalen : Scientia Verlag, 1965, Giessen 1891, p. 9, cf. H. KaLb, Studien zur Summa Stephans von Tournai : ein Beitrag zur kanonistischen Wissenschaftsgeschichte des späten 12. Jabrhunderts, Innsbruck 1983, p. 82). Auteur victorin, éduqué dans la tradition rhétorique française, Étienne connaissait sans doute le commentaire de Guillaume. Malgré tout, les idées laonnoises disparaissent rapidement des gloses des canonistes sur la coutume, ainsi dans les textes rassemblés par R. Weigand, « Das Gewohnheitsrecht in frühen Glossen zum Dekret Gratians ", in : Ius populi Dei. Miscellanea in honorem Raymundi Bidagor, Roma 1972, p. 93-101. 
collectif au Moyen Âge occidental ont conduit à lanarchie ${ }^{122}$. Surtout, la place de l'écrit est sans commune mesure à Beauvais au début du XII ${ }^{\mathrm{e}}$ siècle par rapport à Sumatra au XIX ${ }^{\mathrm{e}}$ siècle. À Saint-Quentin, Yves de Chartres commençait sans doute son travail de compilation et devait avoir à sa disposition une gamme impressionnante de manuscrits canoniques. À une centaine de kilomètres de Beauvais, Maingaud feuilletait peut-être un Code justinien et recevait des informations d'étudiants italiens sur le droit lombard. Le monument du droit écrit occidental disponible aux intellectuels médiévaux est sans commune mesure avec les maigres manuels de shariah que pouvait lire la minorité arabophone des responsables religieux Minangkabau.

\footnotetext{
${ }^{122}$ Du reste, quand les politistes de la fin du Moyen Âge discutent de la question de savoir s'il est préférable d'élire son roi ou que le pouvoir soit transmis de manière héréditaire, ils expliquent que lélection permettrait de choisir le meilleur candidat mais que le risque de troubles est trop grand et que la succession au trône est donc préférable (Ch. de Miramon, "Aux origines de la noblesse et des princes du sang. France et Angleterre au xiv siècle ", in : L'hérédité entre Moyen Âge et Époque moderne. Perspectives historiques, éd. M. van der Lugt/C. De Miramon, Firenze 2008, p. 157-210, p. 191).
} 